\title{
Thermodynamic analysis of the Quantum Critical behavior of Ce-lattice compounds
}

\author{
Julian G. Sereni \\ Div. Bajas Temperaturas, CAB-CNEA, CONICET, 8400 Bariloche, Argentina
}

(Dated: June 3, 2022)

\begin{abstract}
A systematic analysis of low temperature magnetic phase diagrams of Ce compounds is performed in order to recognize the thermodynamic conditions to be fulfilled by those systems to reach a quantum critical regime and, alternatively, to identify other kinds of low temperature behaviors. Based on specific heat $\left(C_{m}\right)$ and entropy $\left(S_{m}\right)$ results, three different types of phase diagrams are recognized: i) with the entropy involved into the ordered phase $\left(S_{M O}\right)$ decreasing proportionally to the ordering temperature $\left(T_{M O}\right)$, ii) those showing a transference of degrees of freedom from the ordered phase to a non-magnetic component, with their $C_{m}\left(T_{M O}\right)$ jump $\left(\Delta C_{m}\right)$ vanishing at finite temperature, and iii) those ending in a critical point at finite temperature because their $\Delta C_{m}$ do not decrease with $T_{M O}$ producing an entropy accumulation at low temperature.

Only those systems belonging to the first case, i.e. with $S_{M O} \rightarrow 0$ as $T_{M O} \rightarrow 0$, can be regarded as candidates for quantum critical behavior. Their magnetic phase boundaries deviate from the classical negative curvature below $T \approx 2.5 \mathrm{~K}$, denouncing frequent misleading extrapolations down to $T=0$. Different characteristic concentrations are recognized and analyzed for Ce-ligand alloyed systems. Particularly, a pre-critical region is identified, where the nature of the magnetic transition undergoes significant modifications, with its $\partial C_{m} / \partial T$ discontinuity strongly affected by magnetic field and showing an increasing remnant entropy at $T \rightarrow 0$. Physical constraints arising from the third law at $T \rightarrow 0$ are discussed and recognized from experimental results.
\end{abstract}




\section{INTRODUCTION}

Magnetic phase diagrams are drown resuming the relevant characteristics of magnetic systems once their basic thermodynamic properties are recognized. Their comparison allows to distinguish between general physical phenomena and the particular behavior of a single compound. The respective magnetic phase boundaries can be traced by applying standard external control parameters like chemical composition $(x)$, pressure $(p)$ or magnetic field $(B)$, able to drive the system into regions of relevant interest. Among them, those related with magnetic instabilities, frustrations, critical points, exotic phases became increasingly attractive because they involve novel phenomena, stressing the understanding of basic physical concepts.

At low temperature, where thermal and quantum fluctuations compete in energy, the neighborhood of quantum critical points (QCP) allows to confront the well established thermodynamical laws with new experimental evidences of quantum effects. In fact, a QCP is currently defined as the $T=0$ limit for a second order transition driven by one of the mentioned non-thermal control parameters [1]. Despite of its unattainable nature at $T=0$, a $\mathrm{QCP}$ carries a sort of halo produced by quantum fluctuations whose physical effects are observed at finite temperature. The phenomenology arising from those low lying energy excitations is usually identified as 'non-Fermi-liquid' (NFL) behavior [2], in contrast to the canonical Fermi-liquid (FL) observed in non-magnetic systems. One of the most relevant features of NFL systems is the increasing density of low energy excitations, manifested as a divergence of thermodynamic parameters like specific heat and thermal expansion divided temperature, and magnetic susceptibility when $T \rightarrow 0$. Accordingly, also electrical resistivity deviates from the $T^{2}$ dependence of a FL showing a typical linear thermal dependence [2. Within this region, the classical magnetic phase transitions (dominated by thermal fluctuations) transform into quantum phase transitions (QPT) gradually dominated by quantum fluctuations [3].

In this work, a comparative analysis of the thermodynamic behavior of Ce-lattice exemplary compounds is carried in order to determine the conditions upon which quantum critical or alternative behaviors can be expected. After to go over the magnetic phase transitions induced by pressure in metallic Ce, an early encompassing phase diagram performed on Ce-binary compounds is reviewed. This phase diagram allowed to recognize some relevant concentrations for Ce-ligand alloyed systems. The second section is devoted to identify different types of entropy evolutions as the ordering temperature decreases because in real systems not all phase boundaries can be extrapolated to zero without basic thermodynamical principles violation. The distinct properties of systems fulfilling the conditions to access to a quantum critical region are presented and discussed in section III, and the thermodynamic implications of the third law approaching the $T \rightarrow 0$ limit are analyzed in section IV. Hereafter, the parameter $T_{M O}$ refers to the order temperature independently of its antiferro (AF) or ferromagnetic (FM) character. However, if any discussed property is only present in AF systems, the phase transition will be identified by the usual Neel temperature $T_{N}$.

\section{A. Ce metal phase diagram}

Despite of the significant progress done during the last decades in the study of low temperature phase diagrams of intermetallic compounds containing $\mathrm{Ce}, \mathrm{Yb}$ and $\mathrm{U}$ lattices [2], some basic phenomena discovered time ago are still under discussion. To our knowledge, the first evidence of anomalous behavior of the $4 f$-electrons was observed nearly eighty years ago when the magnetic behavior of CeN was investigated in 1936 [4. Magnetic measurements showed a weak temperature dependence with a fractional value of the Ce- $4 f$ magnetic moment respect to the expected from the Hund's rule $J=5 / 2$ angular moment. This observation suggested the concept of "intermediate valence" (IV) for the first time in ' $f$ ' elements. Later, on the early sixties, the phase diagram of Ce metal became the subject of a systematic study [5. Driven by pressure, it displays a diversity of phases where structural and magnetic changes are strongly related. The $\gamma \leftrightarrow \alpha$ structural and magnetic phase transition became the fingerprint of the local-itinerant dilemma of Ce- $4 f^{1}$ electrons [6] not yet completely elucidated.

That first order transition is related to the collapse of the Ce atomic volume (about 15\%) and shows an end critical point $(\mathrm{CP})$ at $\approx 600 \mathrm{~K}$ under a pressure of $\approx 2 \mathrm{GPa}$. Within the $\alpha$ structure, a superconductive phase develops up to $T_{s c} \approx 50 \mathrm{mK}$, which jumps up to $T_{s c}=1.9 \mathrm{~K}$ in a second structural collapse between the $\alpha \leftrightarrow \alpha^{\prime}$ phases at $4 \mathrm{GPa}$ [7. This superconductive $4 f$ mediated phase compares in temperature and IV character with the recently highlighted second superconductive dome of $\mathrm{CeCu}_{2} \mathrm{Si}_{2}$ tuned by pressure and claimed to be related to a second QCP in that compound [8]. Since it occurs at the edge of the heavy fermion HF-IV crossover, where the $\mathrm{N}=2$ degeneracy of the HF-ground state (GS) transforms into a $\mathrm{N}=6 \mathrm{GS}$, this critical region can be regarded as a reminiscence of the $\alpha \leftrightarrow \alpha^{\prime}$ transition. Including the CeTIn ${ }_{5}$ compounds [9] and $\mathrm{ZrCe}$ alloys [10], $\approx 2 \mathrm{~K}$ seems to be an upper limit for $T_{s c}$ already detected four decades ago. 

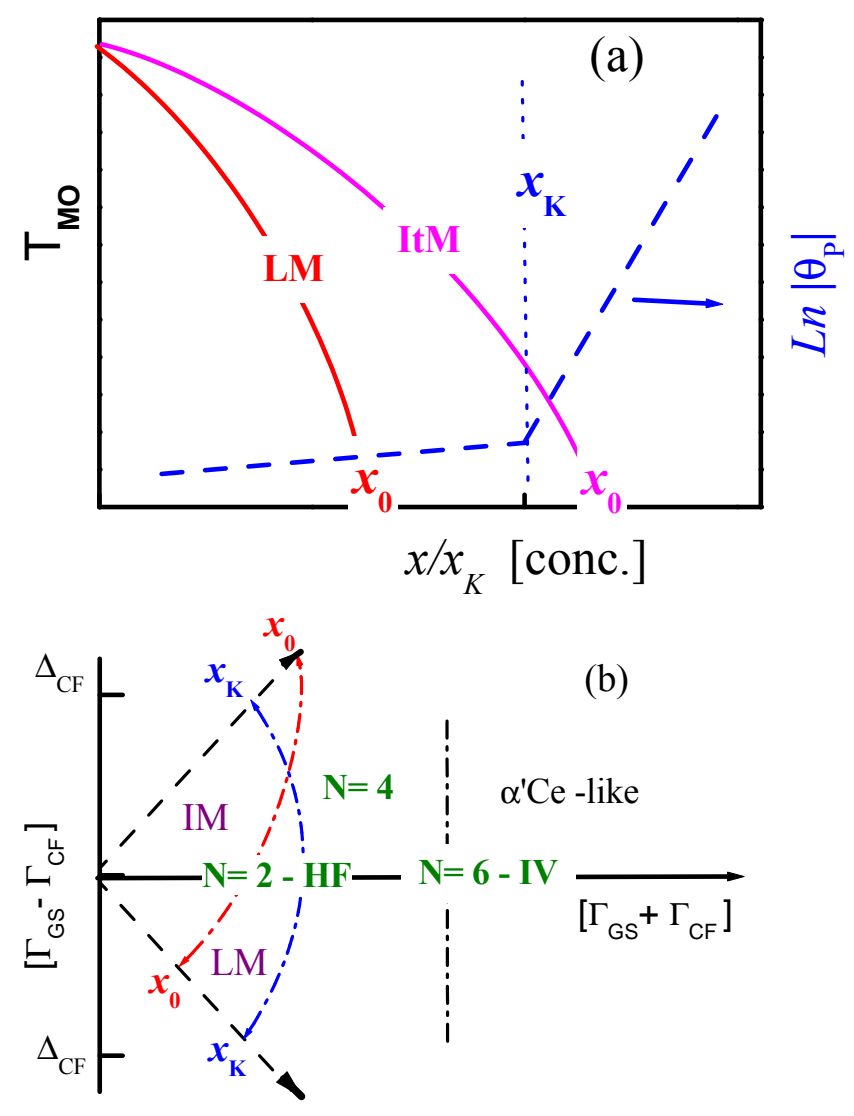

FIG. 1. Schematic magnetic phase diagrams after [13]. (a) $T_{M O}$ ordering temperature, $x_{0}$ concentration of the $T_{M O}(x) \rightarrow 0$ extrapolation, $x_{K}$ onset of $T_{K}(x) \propto \theta_{P}$ increase, LM phase boundary of Ce compounds with local magnetism $\left(x_{0}<x_{K}\right)$ and ItM with itinerant magnetic character $\left(x \geq x_{K}\right)$. (b) Encompassing phase diagram, $\Delta_{C F}$ crystal field splitting, $\Gamma_{G S, C F}$ respective ground and excited levels hybridization strengths, $\mathrm{N}$ different GS degeneracies.

\section{B. Low temperature phase diagrams of Ce-lattice compounds}

A turning-point in the description of $\mathrm{Ce}, \mathrm{Yb}$ and $\mathrm{U}$ magnetic phase diagrams occurred when the competition between on-site Kondo screening and inter-site RKKY interactions was taken into account by theory [11. While the former effect weakens the local $C e-4 f$ moments, the latter provides the long range interaction favoring the magnetic order. The characteristic energies of these mechanisms $\left(k_{B} T_{K}\right.$ and $\left.k_{B} T_{R}\right)$ can be compared through their respective dependencies on the local-conduction band coupling parameter $\left(g=\delta_{F} J_{e x}\right): T_{K} \propto \exp (1 / g)$ and $T_{R} \propto g^{2}$, where $\delta_{F}$ is the density of states and $J_{e x}(<0)$ the usual exchange integral. The nature of the GS is therefore established by the value of $g$, being magnetic $\left(T_{R}>T_{K}\right)$ for small $g$ and non-magnetic $\left(T_{K}>T_{R}\right)$ for large $g$ values. At the intermediate region, where $T_{K}$ and $T_{R}$ are comparable, both mechanisms compete, weakening the local $4 f$ effective moment with the consequent reduction of the ordering temperature $T_{M O}(g)$.

Although this model contains the basic mechanisms which govern the magnetic phase boundaries variation, it is evident that a 'one-parameter' description cannot cover the diversity of behaviors observed applying different control parameters. As example one can mention the different behavior induced by the three usual control parameters: $x, p$ and $H$, on the specific heat of a single sample like $\mathrm{CeCu}_{2}\left(\mathrm{Si}_{0.9} \mathrm{Ge}_{0.1}\right)_{2}$ presente in Fig.15 of Ref. [12]. Furthermore. one may observe different 'trajectories' of the magnetic phase boundaries between the ferromagnetic $\mathrm{CePd}\left(T_{C}=6.5 \mathrm{~K}\right)$ and two IV isotypic compounds $\mathrm{CeRh}$ and $\mathrm{CeNi}$, in both cases driven by alloying the Ce-ligand atom. In the former case: $\mathrm{Ce}\left(\mathrm{Pd}_{1-x} \mathrm{Rh}_{x}\right)$, there is a modification of the chemical potential whereas in the later: $\mathrm{Ce}\left(\mathrm{Pd}_{1-x} \mathrm{Ni}_{x}\right)$, a structural pressure effect [13]. Notice the different effect produced by both control parameters which are usually confused as 


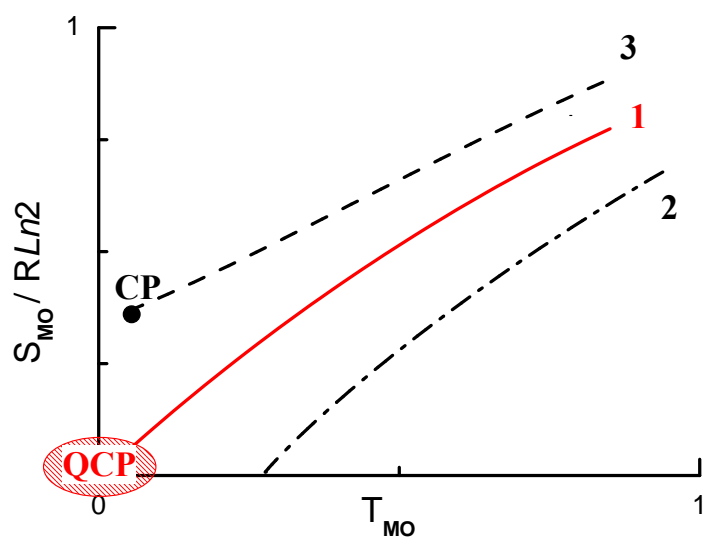

FIG. 2. Schematic representation of three possible dependencies of $S_{M O}\left(T_{M O}\right)$ : 1) (solid line) fulfills the condition to reach a QCP because $S_{M O} \rightarrow 0$ as $T_{M O} \rightarrow 0 ; 2$ ) (dash-dot line) a phase boundary vanishing at finite temperature and 3) (dashed line) a phase boundary ending at a finite temperature critical point $(\mathrm{CP})$, see the text.

being equivalent [14.

\section{Characteristic concentrations in Ce-ligand alloyed systems}

Another limitation for the description of the magnetic phase diagrams using a single parameter dependence arose from the comparative analysis performed on seventeen alloyed Ce-binaries compounds [13. For such analysis, two characteristic concentrations were defined: $x_{0}$, where the magnetic phase boundary $T_{M O}(x)$ extrapolates to $T=0$, and $x_{K}$ where the paramagnetic temperature $\theta_{P}$ starts to rise significantly. It is known that $\theta_{P} \propto T_{K}$ [15] once $\left|\theta_{P}(x)\right|$ becomes much larger than $T_{M O}$. Two distinct types of phase diagrams were identified, one with $x_{0} \geq x_{K}$ and the other with $x_{0}<x_{K}$, which are schematically represented in Fig. 11. Both scenarios correspond to different hybridization strengths $\left(\Gamma \propto T_{K}\right)$ : i) with weak hybridization, i.e. of local moment (LM) character, and ii) with moderate hybridization, i.e. of itinerant (ItM) character [16. This scheme applies to a doublet ( $\mathrm{N}=2$ degenerated) ground state of Ce- $4 f$ ions within the region where $T_{M O}(x)$ decreases. For $x>x_{K}, \Gamma \propto \theta_{P}$ increases more rapidly with the consequent broadening of the magnetic levels. Eventually, an overlap between the ground $\Gamma_{G S}$ and crystal field (CF) excited levels may occur once respective $\Gamma_{G S}$ and $\Gamma_{C F}$ hybridizations strengths become comparable to the CF splitting $\left(\Delta_{C F}\right)$. In the limit of $\Gamma>>\Delta_{C F}$ the IV state, with $\mathrm{N}=6$, takes over.

An encompassing phase diagram was proposed (see Fig. 1 1 ) computing the difference $\left(\Gamma_{G S}-\Gamma_{C F}\right)$ versus the sum $\left(\Gamma_{G S}+\Gamma_{C F}\right)$ of those parameters. According to experimental evidences from the temperature dependence of electrical resistivity $(\rho(T, x))$, the LM regime correspond to systems with $\Gamma_{G S}<\Gamma_{C F}$ and the ItM one to those with $\Gamma_{G S}>\Gamma_{C F}$ 17. Recently, some non magnetic Ce compounds were reported to follow Coqblin-Schrieffer model predictions for a four fold $N=4$ GS $18-20$. Nevertheless, the limit between a $N=4$ GS and a quasi-quartet (i.e. two doublets with small but comparative $\Gamma_{G S}>\Gamma_{C F}$ ) are difficult to be discriminated experimentally.

\section{DIFFERENT TYPES OF MAGNETIC PHASE DIAGRAMS}

With the growing interest on quantum critical phenomena, a number of theoretical models were proposed to describe low lying energy excitations related to the $T_{M O} \rightarrow 0$ physics, see for example Refs. 3, 21 23 and references therein. Since the scope of the models is generally focused on microscopic mechanisms and they are applied on specific exemplary cases, there is an absence of an encompassing criteria able to detect or discard new candidates and, eventually, to recognize new alternative behaviors. Thermodynamic postulates provide the proper tools for such a purpose because of their simplicity and universality. Notably, the constraints imposed by the third law of thermodynamics (e.g. the $T=0$ singularities in thermodynamic parameters) are some times left aside.

Another relevant aspect concerns whether there is any condition to be fulfilled at finite temperature for a real system to actually reach the $T_{M O} \rightarrow 0$ limit. It is evident, for example, that many $T_{M O}(p)$ phase boundaries are not 


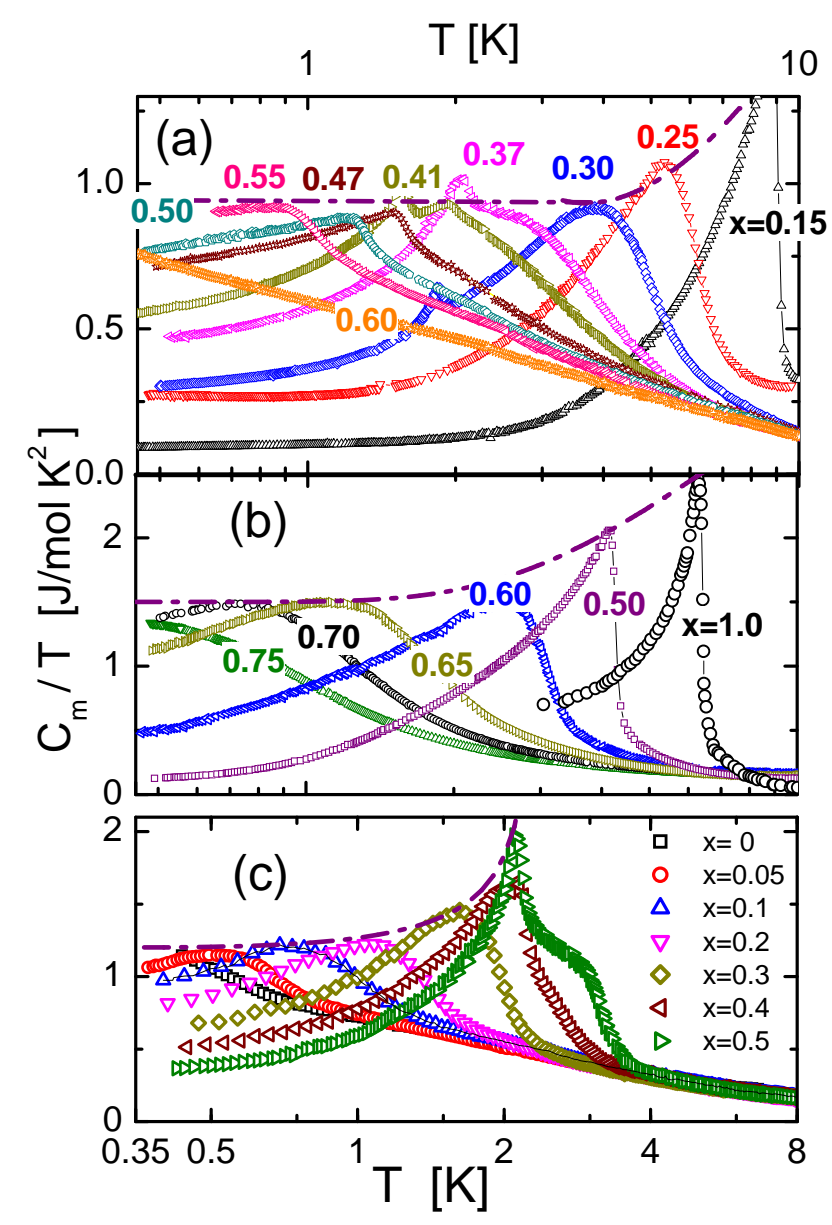

FIG. 3. (Color online) Magnetic contribution to specific heat (after respective phonon subtraction) divided temperature of three exemplary compounds approaching their respective critical regime. (a) $\mathrm{AF}-\mathrm{CeIn}_{3-x} \mathrm{Sn}_{x}$, (b) $\mathrm{FM}_{-\mathrm{CePd}} \mathrm{d}_{1-x} \mathrm{Rh}_{x}$ and (c) AF-CePd ${ }_{2}\left(\mathrm{Ge}_{1-x} \mathrm{Si}_{x}\right)_{2}$ after [25. Dash-dot curves indicate the tendency to a nearly constant value of $C_{m} / T$ maxima within the $x_{0}<x<x_{c r}$ region. Notice the logarithmic $T$ dependence.

properly checked to involve the corresponding entropy of the ordered phase $\left(S_{M O}\right)$ despite their $T_{M O}(P)$ are naïvely extrapolated from $T>1 \mathrm{~K}$ down to e.g. their respective superconductive domes at $T_{s c}<1$. Similar questions apply to concentration driven systems since the arbitrarily monotonous extrapolations of $T_{M O}(x)$ exclude any variation of $\partial T_{M O} / \partial x$, even in the range where thermal and quantum fluctuations start to compete in energy at low temperature.

The amount of intermetallic compounds claimed at present to be candidates for quantum critical behavior allows, and even requires, such a comparative analysis of their thermodynamic properties in order to distinguish between reliable candidates and potential ones. Furthermore, novel alternative behaviors to the quantum phase transitions (QPT) might be missed due to the mentioned simplistic extrapolations done in low temperature phase diagrams.

The simplest starting criterion for such an analysis is to take into account all possible alternative scenarios for the decreasing entropy of the magnetically ordered phase $\left(S_{M O}\right.$, computed from zero up to $\left.T_{M 0}\right)$. There are three possible alternatives: 1) $S_{M O} \rightarrow 0$ as $T_{M 0} \rightarrow 0$;2) $S_{M O} \rightarrow 0$ at finite temperature due to an eventual transference of degrees of freedom to a non-magnetic component, and 3) $S_{M O}$ does not decreases proportionally to $T_{M O}$, producing a entropy accumulation at low temperature. These three cases are schematically represented in Fig. 2 where each case is identified with the respective number.

The exemplary systems selected for this study are Ce-lattice compounds, where composition or chemical pressure effects are produced by alloying Ce-ligand atoms. This criterion preserves the periodicity and local symmetry of the $\mathrm{Ce}$ net in order to minimize side effects such as disorder 24 . Hence, hereafter we will refer to 'alloyed compounds' as those where only Ce-ligands are doped or substituted. Otherwise, the few experimental results extracted from a Ce diluted system will be explicitly indicated. 


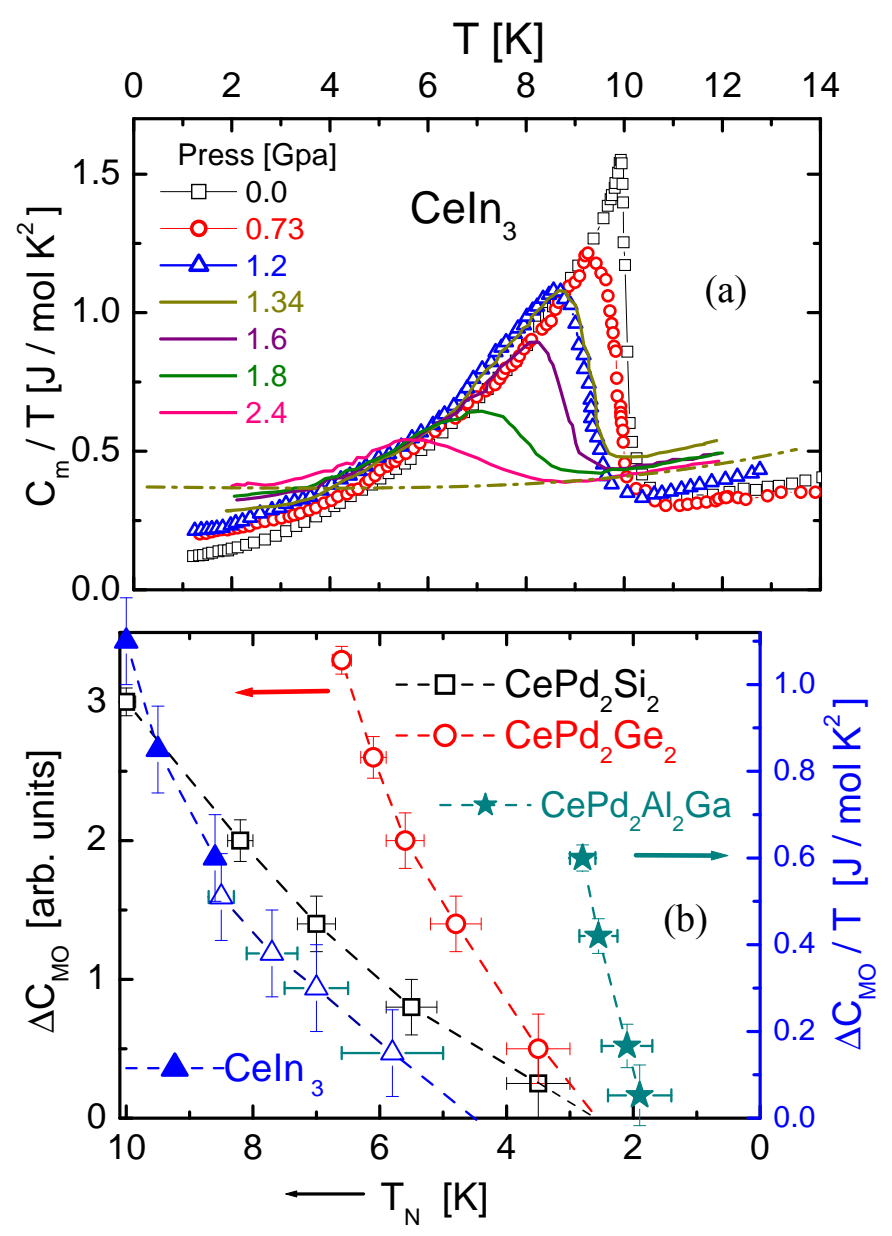

FIG. 4. (Color online) (a) Example of vanishing specific heat jump at finite temperature as a function of pressure after Ref. 32 (open symbols) and Ref. 34] (continuous lines). Dashed curve: non-magnetic HF reference for e.g. 2.4 GPa isobar. (b) Various vanishing specific heat jumps as a function of $T_{N}(p)$. Notice that $T_{N}(p)$ decreases to the right a pressure increases. Open symbols (left axis) extracted from ac-specific heat and full symbols (right axis) from standard heat pulse techniques, with $\mathrm{CeIn}_{3}$ comparing both techniques.

\section{Ce systems with $S_{M O} \rightarrow 0$ as $T_{M} \rightarrow 0$}

This is the thermodynamic condition to be fulfilled for reaching a zero temperature QCP. For this group, we have selected some exemplary Ce-lattice alloyed compounds whose specific heat were measured down to very low temperature, see Fig. 3. The common feature of these experimental results is that the respective specific heat jumps $\left(\Delta C_{m} / T\right)$ at $T_{M O}$ first decrease and then broaden levelling off around $x \approx x_{0}$ [25. This change of regime at $x \approx x_{0}$ is associated to a change of slope of the phase boundary at $x \approx x_{0}$ whose implications are discussed in the following Section. The $C_{m} / T$ maximum for $x \geq x_{0}$ can be analyzed within the Ginzburg-Landau theory for second order transitions: $C_{m} / T=a^{2} / 2 b$, where $a$ and $b$ are the coefficients of the free energy expansion $G(\psi, T)=$ $G_{0}(T)+a(T) \psi^{2}+b(T) \psi^{4}$. The tendency to a constat value of $C_{m a x} / T$ indicates that the $G(\psi, T)$ dependence on the $a^{2} / b$ ratio is locked and consequently the entropy evaluated up to that maximum decreases monotonously, following a sort of law of corresponding states [26] with the critical point at $T=0$. This is a necessary thermodynamical condition for any system to reach a QCP. Based on this analysis, one may include into this group the well known system $\mathrm{CeCu}_{6-x} \mathrm{Au}_{x}$ [27] and $\mathrm{Ce}\left(\mathrm{Pd}_{1-x} \mathrm{Ni}_{x}\right)_{2} \mathrm{Ge}_{2}$ [28] because they exhibit the same fatures. one may even propose a potential candidate like $\mathrm{Ce}\left(\mathrm{Pd}_{1-x} \mathrm{Rh}_{x}\right) \mathrm{In}$ [29] that, to our knowledge, was not yet investigated down to sufficiently low temperature. Among the pressure driven systems, one finds $\mathrm{CePb}_{3} 30$ showing this type of behavior up to $7 \mathrm{GPa}$ where a change of magnetic structure occurs. Since this class of magnetic phase diagrams are directly related to quantum critical phenomena, we shall discuss in detail some selected experimental results in Section III. 


\section{Systems with $S_{M O} \rightarrow 0$ at finite temperature}

A second class of magnetic Ce-lattice systems behave quite differently because $S_{M O}$ decreases faster than $T_{N}$ extrapolating to $S_{M O}=0$ at finite temperature, being all experimental examples AF. This type of behavior is frequently observed in pressure driven stoichiometric compounds and the relevance of this class of phase diagrams arises from the fact that many of them exhibit a superconductive GS under pressure 31.

The appearance of a superconductive dome is currently related to the extrapolation of the AF phase boundary. However, a thermodynamic analysis of those phase boundaries (mostly constructed from resistivity measurements) reveal that such a putative extrapolation of $T_{N}(p)$ down to $T_{s c}$ is quite arbitrary. It is known that to extract absolute values from heat capacity measurements under pressure exceeding $1.5 \mathrm{GPa}$ is quite difficult. Nevertheless, measurements up to $1.2 \mathrm{GPa}$, e.g. in $\mathrm{CeIn}_{3}$ [32] and $\mathrm{CePd}_{2} \mathrm{Al}_{2} \mathrm{Ga}$ [33, are good reference for those performed at higher pressure, e.g. $\mathrm{CeIn}_{3}$ [34, $\mathrm{CePd}_{2} \mathrm{Si}_{2}$ [35] and $\mathrm{CePd}_{2} \mathrm{Ge}_{2}$ [36. These results provide convincing information to recognize their distinct behavior respect to those described in the previous subsection (see Fig. 3). Since very high pressure results are given using arbitrary units, a quantitative evaluation of $S_{M O}(p)$ variation is not possible. Alternatively one may evaluate the relative decrease of the specific heat jump at $T_{M O}\left(\Delta C_{M O}(p)\right)$ taking into account that $\Delta C_{M O} \rightarrow 0$ implies that $S_{M O} \rightarrow 0$.

The common feature of these results is that they show a progressive transference of the magnetic degrees of freedom to a non-magnetic HF component in the region where $T_{N}(p)$ decreases. As an example, the $C_{m}(T)$ results obtained on $\mathrm{CeIn}_{3}$ [32, 34] is presented in Fig. 4a. The comparison with the other compounds is done using the relative variation of $\Delta C_{M 0}$ driven by pressure as depicted in Fig. $4 \mathrm{~b}$. In that figure (right axis) the quantitative comparison result is made using the results from $\mathrm{CePd}_{2} \mathrm{Al}_{2} \mathrm{Ga}[33$.

The main conclusion extracted from Fig. $4 \mathrm{p}$ is that in all these compounds the $\Delta C_{m}\left(T_{N}\right)$ jump vanishes at finite temperature independently of their eventual superconductive GS, with the phase boundary vanishing some degrees of temperature above $T_{s c}$. In the case of $\mathrm{CeIn}_{3}$, this behavior is confirmed by ${ }^{115} \mathrm{In}-\mathrm{NQR}$ measurements 37 under pressure. Detailed electrical resistivity $(\rho)$ measurements performed on $\mathrm{CePd}_{2} \mathrm{Si}_{2}$ [38] also supports this observation since the temperature derivative $\partial \rho / \partial T$ (expected to be qualitatively related to the specific heat [38]) shows a jump at $T=T_{N}$ which also vanishes at finite temperature.

Noteworthy is the fact that these pressure driven superconductors show a similar $T_{N}(0) \times p_{0}=2.9 \pm 0.2 \mathrm{KGPa}$ product [39, being $T_{N}(0)$ the $\mathrm{AF}$ transition temperature at ambient pressure and $p_{0}$ the pressure where the transition vanishes. The origin of this empirical relation is not yet elucidated, but it is confirmed by a number of non-superconductive systems which do not verify that product. Among then, the compounds undergoing a maximum of their phase boundaries as a function of pressure (e.g. $\mathrm{CePd}_{2} \mathrm{Al}_{2} \mathrm{Ga}$ [33] or $\mathrm{CePt}$ [40) clearly do not present a superconductive phase.

It should be mentioned that the competition between magnetism and superconductivity observed in $\mathrm{CeRhIn}_{5} \mathrm{for}$ example [4] cannot be included within this group because its magnetic transition is of first order and there is a coexistence of both phases below $T \approx 2 \mathrm{~K}$ and it merits its own analysis.

Concerning Ce-lattice alloyed systems, one can mention $\mathrm{Ce}\left(\mathrm{Rh}_{1-x} \mathrm{Ru}_{x}\right)_{2} \mathrm{Si}_{2}$ [2], $\mathrm{Ce}\left(\mathrm{Rh}_{1-x} \mathrm{Pd}_{x}\right)_{2} \mathrm{Si}_{2}$ and $\mathrm{Ce}\left(\mathrm{Rh}_{1-x} \mathrm{Ru}_{x}\right)_{3} \mathrm{~B}_{2}$ [12] which show equivalent vanishing process of their magnetically ordered degrees of freedom. Interestingly, two ways of transfer can be distinguished between ItM (large Fermi surface) and LM (small fermi surface) magnetic systems. Within the former group (e.g. Ce( $\left.\left(\mathrm{Rh}_{1-x} \mathrm{Pd}_{x}\right)_{2} \mathrm{Si}_{2}\right)$ the ordered state seems to build up as a condensation of degrees of freedom from the $4 f$ narrow band heavy quasi-particles because an entropy compensation is observed respect to a high temperature $\left(T>T_{N}\right)$ non-Fermi-liquid (NFL) component which does not change with concentration [12. On the contrary, in the second type the transference of degrees of freedom occur between two different components because the NFL component increases at the expense of the exhausting magnetically ordered ones. Preliminary $C_{m}(T)$ results on $\mathrm{Ce}\left(\mathrm{Co}_{1-x} \mathrm{Fe}_{x}\right) \mathrm{Si}$ alloys clearly show this transference between two systems because $\Delta C_{m}\left(T_{N}\right)$ vanishes around $T \approx 4.5 \mathrm{~K}$ with a coincident rising up of a $C_{m} / T \propto-\operatorname{Ln}\left(T / T_{0}\right)$ contribution. No superconductive GS is expected in these alloyed systems because of the Ce neighbors random distribution. Nevertheless, the non-magnetic stoichiometric limit $(x=1)$ of $\mathrm{Ce}\left(\mathrm{Rh}_{1-x} \mathrm{Ru}_{x}\right)_{3} \mathrm{~B}_{2}$ shows low temperature superconductivity [43.

\section{Systems with critical entropy accumulation as $T_{N}$ decreases}

According to thermodynamics, if $S_{M O}$ does not decrease proportionally to $T_{M O}(x)$ (like in the mentioned first class) but with lower ratio, a $\lim _{T \rightarrow 0} S_{M O}>0$ would occur. In that case, an entropy bottleneck occurs and the magnetic phase boundary shall end in a CP at finite temperature where a first order transition drives the system to $S_{M O} \rightarrow 0$. Such a situation is observed in the compounds included in Fig. 5 . $\mathrm{Ce}_{2}\left(\mathrm{Ni}_{1-x} \mathrm{Pd}_{x}\right)_{2} \mathrm{Sn}$ [4] and $\mathrm{URu}_{2} \mathrm{Si}_{2}$ [45]. The former is a recently studied compound driven by Ce-ligands alloying, whereas the latter is the well known 

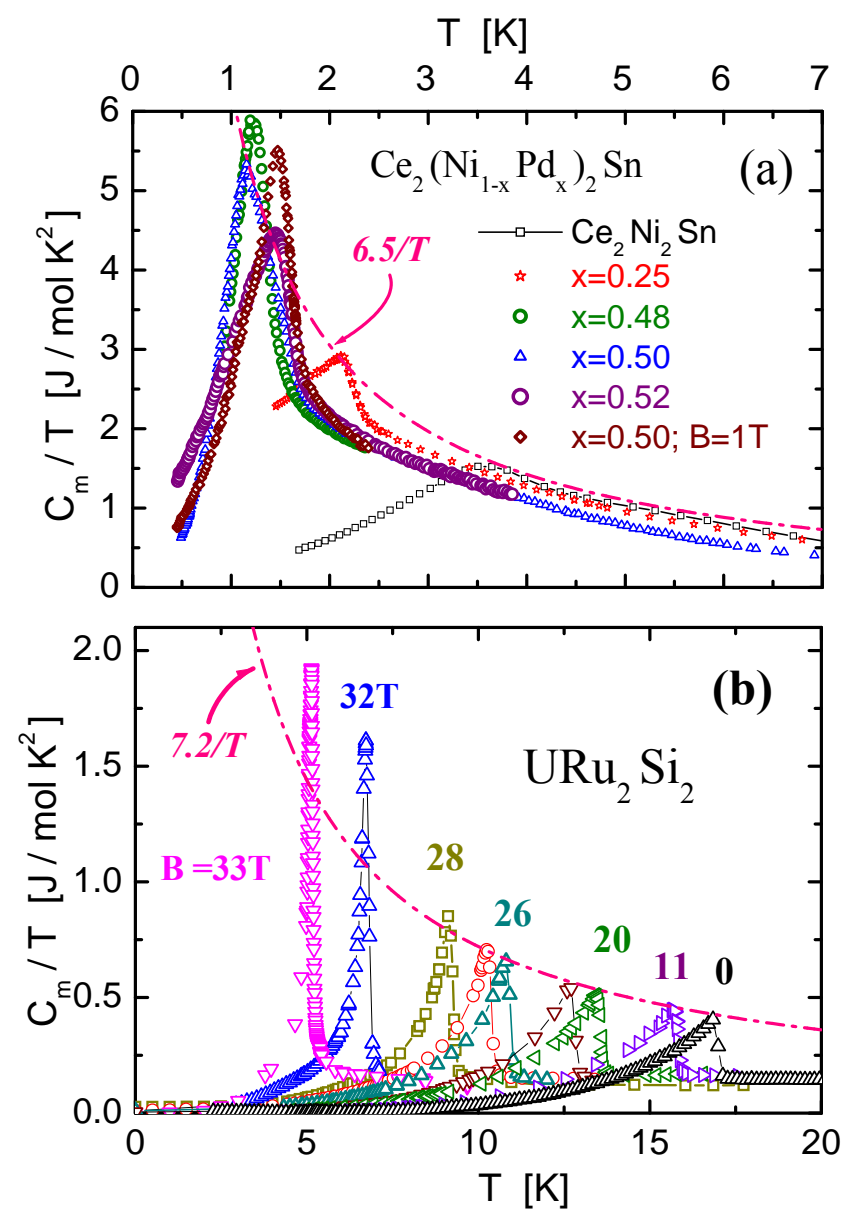

FIG. 5. (Color online) (a) Specific heat divided temperature of the concentration dependent $\mathrm{Ce}_{2}\left(\mathrm{Ni}_{1-x} \mathrm{Pd}_{x}\right)_{2} \mathrm{Sn}_{\mathrm{s}} \mathrm{s}$ stem after Ref. 44] and (b) referent field dependent $\mathrm{URu}_{2} \mathrm{Si}_{2}$ after Ref. 45]

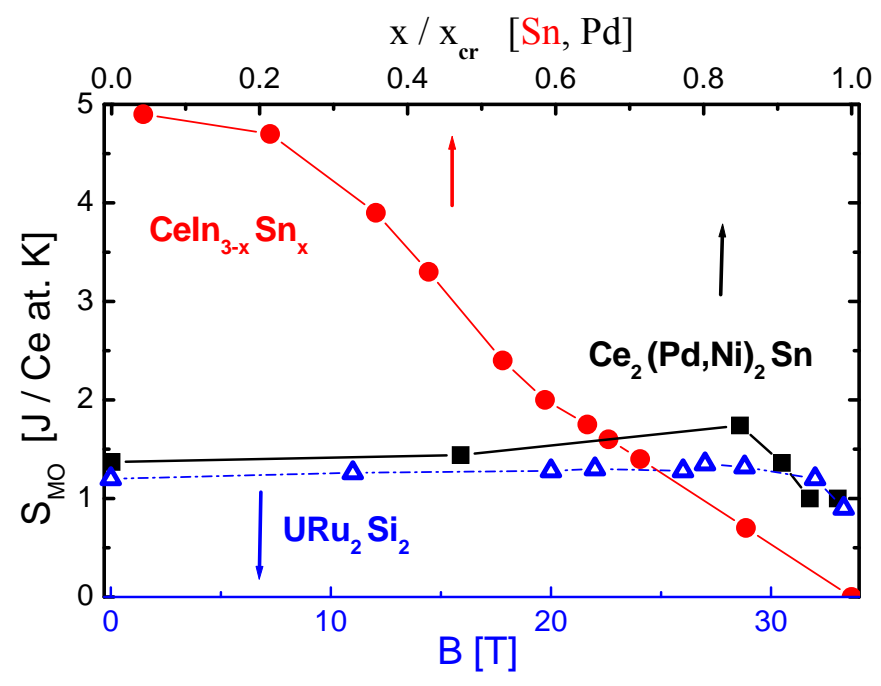

FIG. 6. (Color online) Comparison of the entropy gain $S_{M O}$ up to $T_{N}$ between two types of behaviors: CeIn ${ }_{3-x} \mathrm{Sn}_{x}$ of the $1 \mathrm{st}$. class and $\mathrm{Ce}_{2}\left(\mathrm{Ni}_{1-x} \mathrm{Pd}_{x}\right)_{2} \mathrm{Sn}$, and $\mathrm{URu}_{2} \mathrm{Si}_{2}$ of the $3 \mathrm{rd}$. class. Upper x-axis for concentration $x$ dependent systems, and the lower $\mathrm{x}$-axis for the magnetic field dependent one. Notice that $\mathrm{Ce}_{2}\left(\mathrm{Ni}_{1-x} \mathrm{Pd}_{x}\right)_{2} \mathrm{Sn}$ contains two Ce-at. per formula unit. 
$\mathrm{U}$ compound driven by magnetic field and taken as a referent system for our purpose. It is worth to note that the $C_{m}(x$ or B $) / T$ variation of the maxima are described by the same function: 6.5 and $7.2 / T$ respectively, both drown in Fig. 5. In contrast to the behavior discussed in subsection II-1 where $C_{m} / T_{\max }$ becomes constant, in this case is the $C_{\max }\left(T_{N}\right)$ value that remains nearly constant till the first order transition occurs (at $B \approx 33 \mathrm{~T}$ in $\mathrm{URu}_{2} \mathrm{Si}_{2}$ ). The first order character of the transitions are recognized from the value of the $C_{m}\left(T_{N}\right)$ maximum which clearly exceed the $\propto 1 / T$ function.

There are also striking coincidences in $S_{M O}$ concerning their similar and nearly constant values that can be appreciated in Fig. 6. The fact that different control parameters applied on different systems produce the same effects can be taken as a fingerprint for the universality of this behavior. For comparison, in Fig. 6 the $S_{M O}$ values obtained from $\mathrm{CeIn}_{3-x} \mathrm{Sn}_{x}$, which belongs to the first group, are also included to show that approaching the critical region the entropy of these two compounds exceed that of one following the low of corresponding states with a $\mathrm{CP}$ at $T=0$.

\section{PECULIAR PROPERTIES OF CE-LATTICE SYSTEMS ACCESSING TO $S_{M O} \rightarrow 0$}

At the time when the phase diagram presented in Fig. 1 was proposed, no quantum fluctuation effects were yet identified and the usual low temperature limit for magnetic studies $\approx 1 \mathrm{~K}$ didn't provide evidences for such a scenario. Thus, the $T_{M O}(x) \rightarrow 0$ limit was usually extrapolated following the classical negative curvature to the concentration defined as $x_{0}$ in Fig. 1. Later on, lower temperature measurements made evident that approaching $x_{0}$ a change of curvature (as presented in Fig. 7] occurs around $T^{C R} \approx 2.5 \mathrm{~K}$ [47. Hence, the actual quantum critical concentration $x_{c r}$ does not coincide with the $T_{M O}$ extrapolation to $x_{0}$ but it occurs at higher values of $x$. Nevertheless, $x_{0}$ remains a relevant concentration because it characterizes the high temperature region dominated by classical thermal fluctuations. To our knowledge, this change of slope was not observed in phase boundaries driven by pressure nor by magnetic field. However, there is a striking coincidence in the fact that the phase boundaries driven by pressure vanish at similar or higher temperatures than $T^{C R}$.

Apart from the mentioned modification of the $T_{M O}(x)$ curvature, the change of regime at $x_{0}$ coincides with other unpredicted features occurring around that concentration. Among them, there is a first order transition $T_{I}(x)$ observed the compounds included in Fig. 3, c.f. $\mathrm{AF} \mathrm{CeIn}_{3-x} \mathrm{Sn}_{x}$ [48, and $\mathrm{CePd}_{2} \mathrm{Ge}_{2-x} \mathrm{Si}_{x}$ [9] and $\mathrm{FM} \mathrm{Ce}\left(\mathrm{Pd}_{1-x} \mathrm{Rh}_{x}\right)$ single crystals [50. The exemplary case of $\mathrm{CeIn}_{3-x} \mathrm{Sn}_{x}$ is depicted in Fig. 8 and will be discussed in detail in Section III-B. Other Ce systems showing a satellite first order transition in similar context are $\mathrm{Ce}\left(\mathrm{Pd}_{1-x} \mathrm{Rh}_{x}\right) \operatorname{In}[29$ and $\mathrm{Ce}\left(\mathrm{Cu}_{1-x} \mathrm{Ni}_{x}\right)_{2} \mathrm{Ge}_{2}$ [51. All these transition shows a similar sharpness, only depending of the quality of the sample.

\section{A. Magnetic Phase Boundary within the Quantum Critical regime}

The observed change of regime can be explained taking into account the competition between the decreasing energy of thermal fluctuations and the temperature independent energy of quantum fluctuations. While the classical transition extrapolates to $x=x_{0}$ with coherent thermal fluctuations of the order parameter decreasing with temperature, the critical fluctuation associated to a QPT respond to a quantum-statistical description [3. As mentioned before, only composition driven phase diagrams show this clear change of regime at $T^{C R} K$ (see Fig. 7 p). In composition driven systems, the formation of static disorder fluctuations (or "rare regions" [1) was proposed to explain the shift of the critical regime from $x_{0}$ to $x_{c r}$.

The occurrence of "rare regions" together with the so-called Griffiths effects [3] are mostly addressed to describe composition driven systems because of their intrinsic possibility of local disorder attributed to all alloyed systems. As it was discussed in Subsection I-C, there is a clear difference between structural and chemical potential pressure effects because the former implies a random (i.e. disordered) Ce-ligand atomic volume distribution. This effect cannot be naïvly extrapolated to chemical potential variation introduced by neighbor elements with nearly equal atomic volume substitution. This effect is therefore better described as an electronic topological distribution. In fact, the change of regime occurs: i) quite suddenly and only close to $x_{0}$, ii) in different compounds with quite different relative concentrations and iii) without a further broadening at higher concentrations [52. Moreover, the first order transitions at $T_{I}$ (see e.g. inset in Fig. 8), show similar sharpness in all cases despite of their different concentration regions excluding any atomic disorder as a relevant factor.

All these experimental evidences confirm that there is a crossover between two distinct regimes at similar temperature. Beyond that region, QPT mechanisms dominate the low energy scenario producing drastic changes in the nature of the magnetic phase boundary as it will be analyzed in detail in the following subsection. Some of those effects can be observed in Fig. 7b for two AF compounds where $T_{M} \propto\left|x-x_{c r}\right|$ and in Fig. 7c for a FM one, where $T_{C}(x)$ decreases asymptotically till it collapses to zero at very low temperature. Notice that for the $x>x_{0}$ region the phase boundary is label as $T_{M}$ instead of $T_{M O}$ in order to distinguish them as belonging to different regimes. If one 


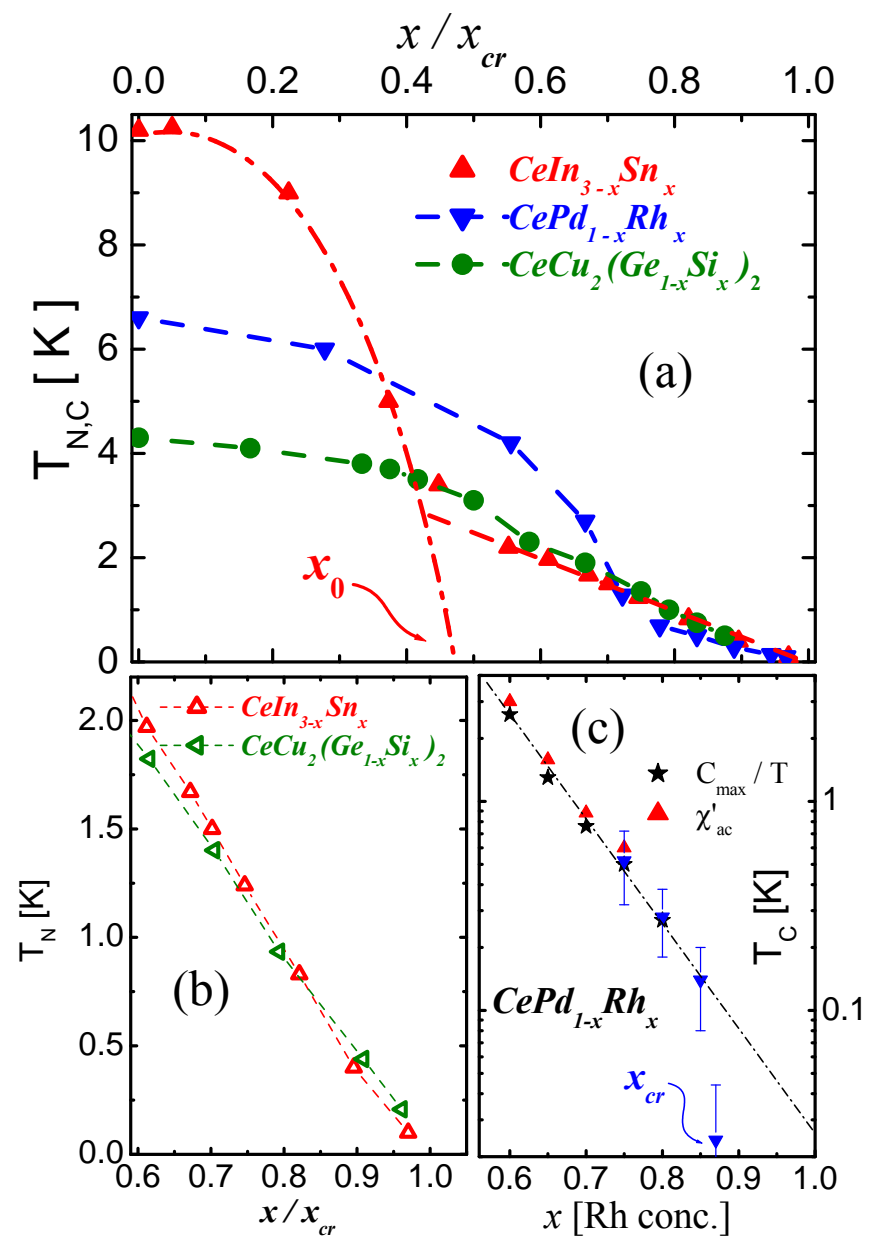

FIG. 7. (Color online) (a) Magnetic phase diagrams of three exemplary compounds covering an extended range of temperature. (b) and (c) show the detail of the quantum critical region for: (b) two AF compounds with linear $T_{N}(x)$ dependence and (c) with an asymptotic phase boundary of a FM compound [46] (notice the logarithmic $T_{C}$ axis).

describes these magnetic phase diagrams within the pattern proposed in Fig. 1. one may recognize that the systems included in Fig. 7b belong to the $x_{0}<x_{K}$ class whereas that from Fig. 7f to the $x_{0} \geq x_{K}$ ones.

To gain insight into this unexplored range of concentration between $x_{0} \leq x \leq x_{c r}$, we will address our attention to the phase diagram and the related thermal properties of $\mathrm{CeIn}_{3-x} \mathrm{Sn}_{x}$. In Fig. 8 we shown a detail of the phase diagram around the critical region where three phase boundaries converge: i) the classical $\mathrm{AF}-T_{N}(x)$ dominated by thermal fluctuations, ii) $T_{M}(x)$ dominated by quantum excitations, and iii) the dome of a first order transition $T_{I}(x)$ curve. The different nature of $T_{M}$ respect to the classical AF-transition at $T_{N}\left(x<x_{0}\right.$ can be clearly appreciated in the inset of Fig. 8 from the flattening of the transition by magnetic field (up to $B=4 \mathrm{~T}$ ) measured on sample $x=0.45$. The same behavior is observed at higher concentration down to the milikelvin temperature range 54 . As a comparison, one can mention that magnetic field applied to an $x=0.25<x_{0}$ sample [55] shows that a very high field (about $40 \mathrm{~T}$ ) has to be applied to flatten the $T_{N}$ transition, which then decreases in temperature before to vanish. Coming back to the studied $x=0.45$ sample, the jump at $T_{M}$ can only be observed analyzing the derivative of $C_{m}(T)$ as shown in the lower part of that inset. To our knowledge, the CR scenario presented in Fig. 8 was only recently pointed out by theory for itinerant FM systems [56. However, our observations are also include quite localized AF systems. Although a first order dome occurs close to CR similarly as proposed by theory, the following $T_{M}(x)$ transitions resamble Pippard's third order ones [57. 


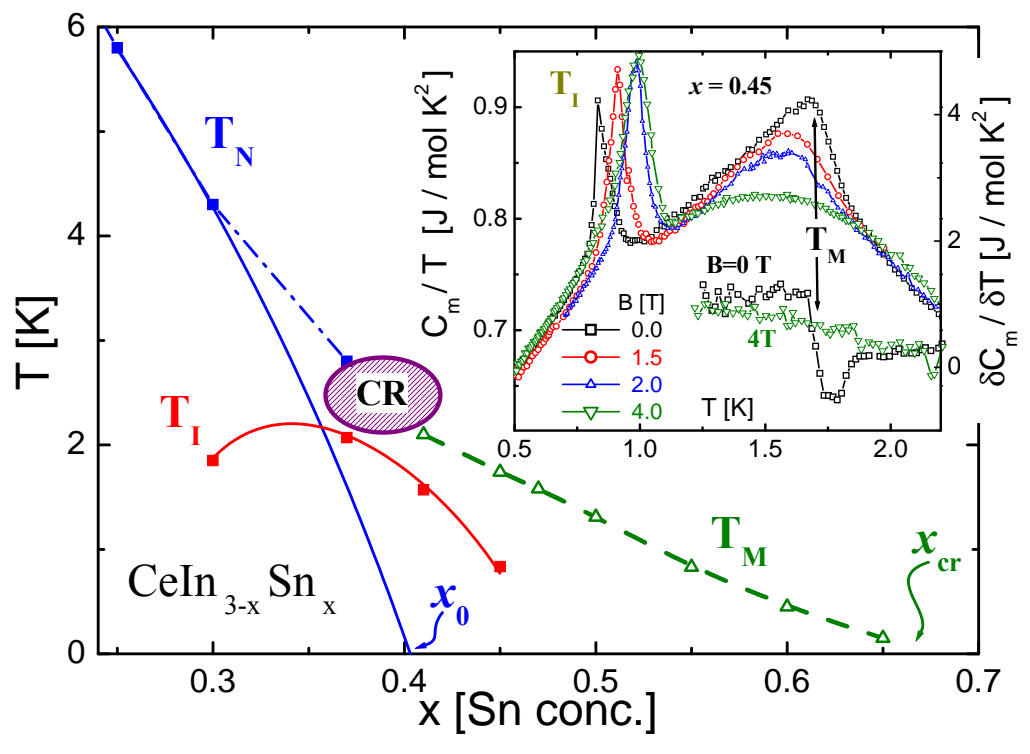

FIG. 8. (Color online) Detailed phase diagram of $\mathrm{CeIn}_{3-x} \mathrm{Sn}_{x}$ around the critical region CR. One can identify different phase boundaries: second order transitions $T_{N}$ for $x \leq 0.3$, a first order dome $T_{I}$ around CR $(0.3 \leq x \leq 0.45)$ and the $T_{M}$ boundary within the pre-critical region $\left(x_{0} \leq x \leq x_{c r}\right)$. Inset: Magnetic field effect on $T_{M}$ and $T_{I}$ transitions on $\mathrm{sample}^{\mathrm{CeIn}} 2.55 \mathrm{Sn}_{0.45}$ after Ref. [48]. Right axis (lower curves) : suppression of the $C_{m}\left(T_{M}\right)$ derivative jump for $B=4 \mathrm{~T}$.

\section{B. Thermodynamic behavior of $\operatorname{CeIn}_{3-x} \operatorname{Sn}_{x}$ within the $x_{0}<x<x_{c r}$ range}

In this subsection we will analyze the $C_{m}(T)$ and entropy $\left(S_{m}(T)\right)$ contributions of the $\mathrm{CeIn}_{3-x} \mathrm{Sn}_{x}$ system, which was investigated in detail within the $x_{0}<x<x_{c r}$ range [48. Taking profit of the linear variation of $T_{M}$ with $x$, one can compare the $C_{m}(T) / T$ dependence of different samples by normalizing the temperature as $\Delta T(x)=T-T_{M}(x)$. In Fig. 9 we show that comparison performed on seven samples covering the concentration range between $0.41 \leq x \leq 0.80$. Notice that with this definition of $\Delta T$, the magnetically ordered phase corresponds to the negative range of that parameter, see the upper x-axis of Fig. 9 . There, one can see how the $C_{m}\left(T>T_{M}\right) / T$ tails of the alloys belonging to the $0.45 \leq x \leq 0.70$ range converge into a unique curve. In order to remark the validity of this scaling, we have also included in the figure the results obtained from $x=0.41$ and 0.80 samples, placed beyond the limits of the pre-critical region, which clearly deviate from the scaled ones.

In Fig. 9 the vertical line at $\Delta T=0$ line splits the $C_{m} / T$ contribution into two parts, the $T<T_{M}$ one (hereafter label as $\left.C_{M} / T\right)$ and the tail at $\Delta T>0$ hereafter identified as $C_{N F L} / T$ because of its NFL behavior (c.f. $\propto \operatorname{Ln}\left(T / T_{0}\right)$ ). Notably, also the $C_{M} / T$ contribution for the samples within this pre-critical region overlap each other at $\Delta T<0$. Samples $x=0.41$ and 0.45 show the weak peak related to a first order transition discussed in the previous subsection. The relevant feature is that the $C_{M} / T$ overlap allows an extrapolation of $C_{M} / T \rightarrow 0$ to $\Delta T \approx-4 \mathrm{~K}$ which is also independent of concentration. We remind that a $\Delta T<0$ value does not correspond to a negative temperature but simply to a common extrapolation to a zero value of the $C_{m} / T$ contribution.

The key parameter to describe this peculiar behavior of the specific heat is its associated entropy, evaluated as $S_{m}=\int C_{m} / T d T$. According to the definition proposed for $\Delta T$, one may split the total measured values as $S_{m}=$ $S_{M O}+S_{N F L}$, being $S_{M}$ the contribution of the $T<T_{M}$ phase (i.e. $\Delta T \leq 0$ ) and $S_{N F L}$ the one from the NFL tail for $\Delta T \geq 0$. For the following analysis we take as reference the entropy variation of sample $x=0.41$ because it contains largest $S_{M}$ contribution among the samples included in Fig. 9. As it can be appreciated in the figure, the $S_{M}(x=0.41)$ contribution slightly exceeds $0.2 R L n 2$ whereas $S_{N F L}$ reaches $\approx 0.6 R L n 2$ (see inner right axis). Noteworthy, the full $R L n 2$ value is only reached once the extrapolation to the $C_{m} / T=0$ value at $\Delta T \approx-4 \mathrm{~K}$ is included, as depicted using the lowest ' $\Delta T+4$ ' and outer ' $S_{M}+S_{N F L}$ ' right axes in Fig. 9 . Since $S_{N F L} \approx 0.6 R L n 2$ does not change with concentration, but $S_{M} \rightarrow 0$ as $x \rightarrow x_{c r}$, one concludes that about $40 \%$ of the $R \operatorname{Ln} 2$ entropy is missed as $T_{M} \rightarrow 0$. that difference is illustrated in Fig. 10a by comparing the $S_{m}(T)$ variation from samples $x=0.15$ (with full entropy) and $x=0.70 \approx_{c r}$.

It is evident from Fig. 9 that the decrease of $S_{M}\left(x \rightarrow x_{c r}\right)$ does not imply a transference of entropy to the NFL phase because $S_{N F L}$ is independent of concentration within this concentration range. Consequently, those degrees of freedom are missed without any change of $S_{N F L}$, which remains unchanged with a $60 \%$ of the $R L n 2$ value. We 


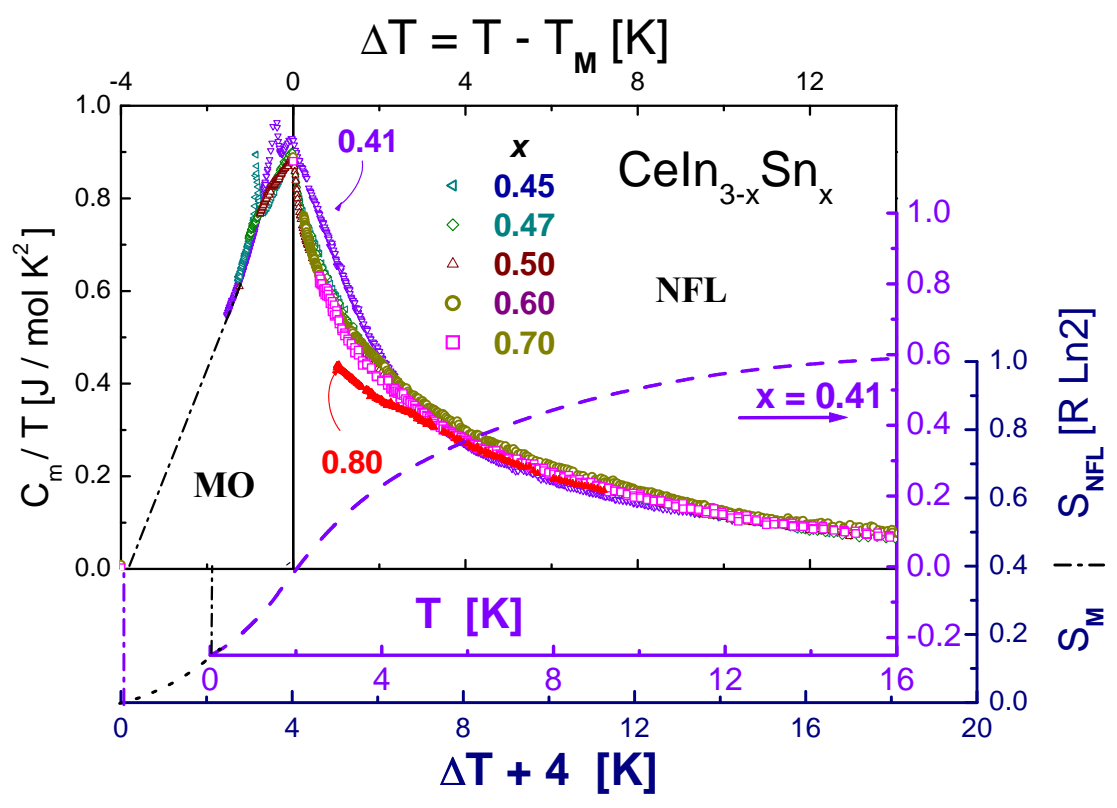

FIG. 9. (Color online) Overlap of $C_{m} / T(0.45 \leq x \leq 0.70)$ curves plotted versus a shifted temperature $\Delta T=T-T_{M}$ (upper and left axes) after Ref.[25. The dashed curve represents the concentration independent entropy gain for $0.45 \leq x \leq 0.70$ samples, discriminated between the $T<T_{M}$ range $S_{M}$ and the paramagnetic one $S_{N F L}$ (low $T$ and inner $S$ axes). Full $R L n 2$ entropy is computed from $\Delta T=-4 \mathrm{~K}$ (lowest ' $\Delta T+4$ ' and outer ' $S_{M}+S_{N F L}$ ' right axes), see the text.

resume this situation in Fig. $10 \mathrm{~b}$, where measured $S_{m}$ up to $T=20 \mathrm{~K}$ and $S_{0}=R \operatorname{Ln} 2-S_{m}$ are represented as a function of concentration. At the QCP, $S_{m} \rightarrow S_{N F L}$ because $S_{M} \rightarrow 0$ and $S_{0} \rightarrow 40 \% R L n 2$.

Simplistic explanations looking for a some extra entropy contribution at higher temperature fail because it would imply a discontinuous transference of entropy from the $T<T_{M}$ to temperatures above $20 \mathrm{~K}$ according to Fig. 9. We recall that in $\mathrm{CeIn}_{3}$ the crystal-field excited quartet lies at high enough energy $(\approx 100 \mathrm{~K}[58$ ) which guaranties no contribution to this analysis. Neither a Kondo temperature increase can be argued because the temperature of the maximum of the electrical resistivity $\left(T_{\max }^{\rho}\right)$ remains unchanged between $x_{0}$ and $x_{c r}$ at $T_{\max }^{\rho} \approx 19 \mathrm{~K}[48$.

The lack of entropy showed by this Ce compound is not an exception because in the cases where this type of analysis was performed the RLn2 value for a doublet GS was never reached. For example, the compounds showing a $C_{m} / T \propto \operatorname{Ln}\left(T / T_{0}\right)$ dependence only reach a $0.54 R L n 2$ value [59]. Since the mentioned $\operatorname{Ln}\left(T / T_{0}\right)$ dependence corresponds to one of the possible scenarios for QCPs predicted by theory [3], one infers that this deficit in the entropy or the consequent arising of remnant entropy at $T=0\left(S_{0}\right)$ is intrinsic to the NFL phenomenology approaching that point.

The question arises whether the low temperature $x \rightarrow x_{c r}$ behavior is governed by low dimensional fluctuations as it was observed in $\mathrm{CeCu}_{5+x} \mathrm{Au}_{1-x}$ [60. Unfortunately neutron scattering studies on $\mathrm{CeIn}_{3-x} \mathrm{Sn}_{x}$ are not available because of the strong neutron absorption of In nuclei. Alternatively, one may check whether any evidence for low dimensional fluctuations can be extracted from thermodynamical results. For that purpose one can evaluate the internal energy, $U_{m}(x)$ and $S_{m}(x)$ for $T_{M}<T<\infty$ in samples around that concentration, and compare them with Ising and Heisenberg model predictions [61] for 1, 2 and 3 dimensional systems with different lattice structures (i.e. coordination number). Such analysis showed that the those thermodynamic parameters nicely fit into the predicted values for a $2 \mathrm{D}$ - Ising quadratic layer [62].

\section{Thermal Expansion}

In order to confirm that the anomalous evolution of the entropy approaching the critical point as due to an intrinsic effect, a complementary thermodynamic parameter sensitive to this phenomenon has to be investigated. Such alternative is provided by thermal expansion $\beta(T, x)$ measurements because they are related to the entropy through the Maxwell relation $-\partial S / \partial P=\partial V / \partial T=\beta$. Thus an anomalous $S_{0}\left(x \rightarrow x_{c r}\right)$ dependence should have a replica in $V_{0}\left(x \rightarrow x_{c r}\right)$ as $T \rightarrow 0$. In this case, the effective pressure is originated in the chemical pressure produced 


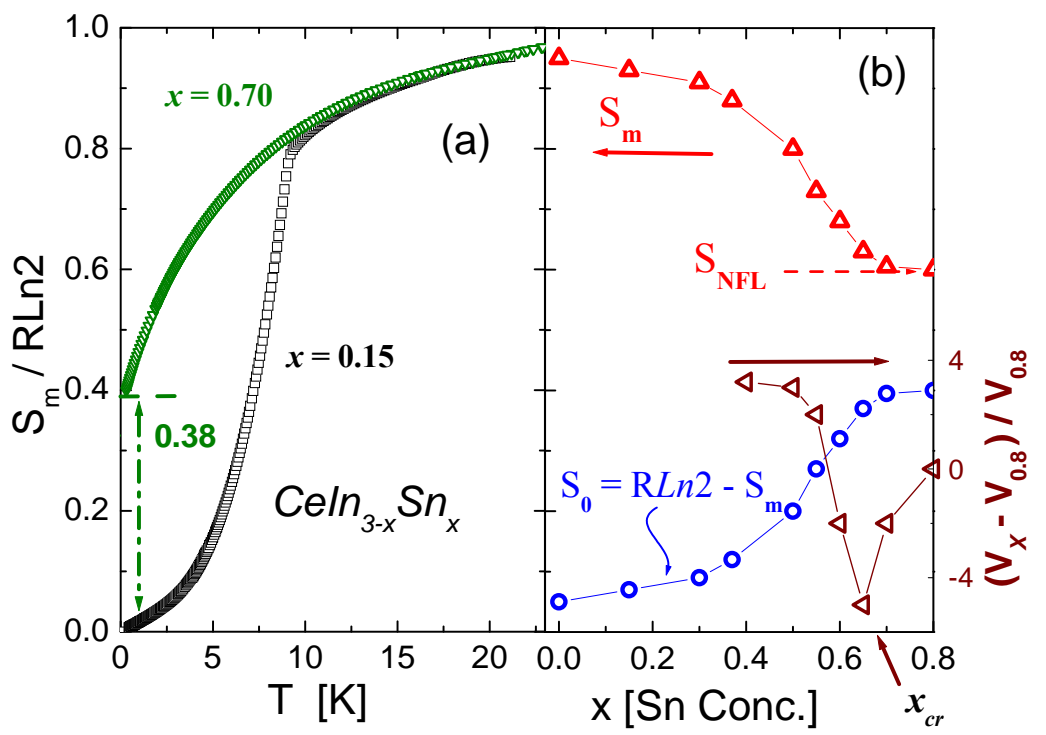

FIG. 10. (Color online) a) Comparison of the temperature dependence of $S_{m}$ between the alloy $x=0.15$ belonging to the classical region and one lying on top of the critical concentration $x=0.70$ showing a deficit of $\approx 40 \%$. b) Left axis: concentration dependence of the entropy $S_{m}=S_{M}+S_{N F L}$ of CeIn ${ }_{3-x} \operatorname{Sn}_{x}$ samples measured up to 20K, and the zero point entropy $S_{0}$ computed as the difference respect the total expected value $\mathrm{R} L n 2 . x_{c r}$ highlights the critical concentration. Right axis: relative change of volume at $T \rightarrow 0$ for $x \geq 0.4$ samples, see text.

by alloying.

The $\beta(T \rightarrow 0, x)$ variation of $\mathrm{CeIn}_{3-x} \mathrm{Sn}_{x}$ was studied down to the $\mathrm{mK}$ range in the vicinity of the critical concentration 63. In Fig. 10p we have included the volume variation as $V_{0}(x)$ for $T \rightarrow 0$ extracted from the temperature variation $V(T)=\sqrt{\beta} d T$. The obtained values were normalized well above any quantum fluctuation effect, i.e. $4 K \leq T \leq 8 K$ taking as reference the $x=0.8$ alloy which lies beyond the critical point. Both abnormal $S_{0}(x)$ and $V_{0}(x)$ dependencies are compared in Fig. 10p around $x_{c r}$.

\section{THERMODYNAMIC IMPLICATIONS OF THE $T \rightarrow 0$ PHYSICS}

\section{A. Third law of Thermodynamics and Remnant Entropy}

Thermodynamic postulates [26] state that the entropy decreases as $T \rightarrow 0$ reaching a finite value $S_{0}$, which is not necessarily zero [57. The implication of this postulate can be viewed in a different way by considering the thermodynamic definition of temperature [26], i.e. that $T=0$ is reached once $\partial U / \partial S=0$. Thus, the thermodynamic condition for zero temperature corresponds to a zero variation of the internal energy $U$ and not to the value of the entropy itself. The $S_{0}=0$ value corresponds to a singlet GS without any other accessible degree of freedom [64. Meta-stable states may eventually decay into such a GS in infinite time (like e.g. amorphous or other structurally disordered systems), however frustrated systems or those dominated by quantum fluctuations (like those involved in the present study) escape to this possibility.

The way to prove that $S_{0} \neq 0$ was applied in Section III-C by taking as reference the $S_{m}=R L n 2$ value associated to the magnetic doublet-GS of $\mathrm{CeIn}_{3-x} \mathrm{Sn}_{x}$ at $T \approx 20 \mathrm{~K}$. Interestingly, the absolute reference for the the entropy value is taken form high enough temperature where both levels of the doublet GS are equally occupied (i.e. $S_{m}=R L n 2$ ).

A complementary aspect regarding the application of the third law of thermodynamics implies the $\lim _{T \rightarrow 0} \partial S / \partial T$. The $\partial S / \partial T=0$ possibility at $T=0$ corresponds to the previously mentioned case of a singlet GS, realized in a long range ordered state and associated to a positive $S_{m}(T)$ curvature (i.e. $\partial^{2} S_{m} / \partial T^{2}>0$ ). The simplest examples for a $\partial S / \partial T \neq 0$ ( or $\left.\partial^{2} S_{m} / \partial T^{2}=0\right)$ is provided by metallic systems whose conduction electrons are described by standard Fermi-liquid behavior with $\partial S / \partial T=\gamma$, c.f. the Sommerfeld coefficient. Heavy fermion (HF) systems simply increases the $\partial S / \partial T$ slope without changing their physical properties.

Since a negative $S_{m}(T \rightarrow 0)$ curvature (i.e. $\left.\partial^{2} S_{m} / \partial T^{2}<0\right)$ is not possible because it would imply a singularity at $T=0$, the question arises whether there is an upper limit for the $\partial S / \partial T=C_{m} / T$ slope or, equivalently, for the 


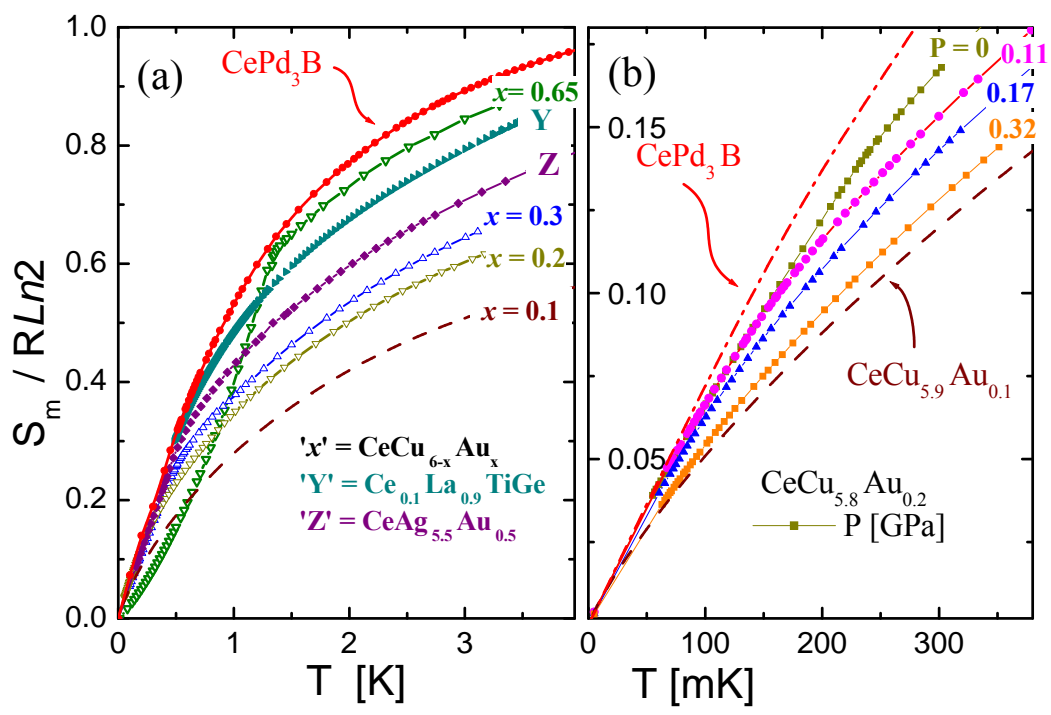

FIG. 11. Examples of maximum slope of $S_{m}(T)$ at $T \rightarrow 0$ in real systems. (a) $\mathrm{CePd}_{3} \mathrm{~B}$ [68, $\mathrm{CeCu}_{6-x} \mathrm{Au}_{x}$ [27? ], $\left(\mathrm{Ce}_{1-y} \mathrm{La}_{y}\right)$ TiGe [66] and $\mathrm{CeAg}_{6-z} \mathrm{Au}_{z}$ [65. (b) Detail at the mK range including: $\mathrm{CeCu}_{5.9} \mathrm{Au}_{0.1}\left(\right.$ dashed line), $\mathrm{CeCu}_{5.8} \mathrm{Au}_{0.2}$ at different pressures and, for comparison, the fit for $\mathrm{CePd}_{3} \mathrm{~B}$ from $T>0.5 \mathrm{~K}$ (dot dashed line).

$\gamma$ value in non ordered HF. Such a question is related to the divergence of thermal parameters like specific heat or thermal expansion because even a logarithmic divergence at $T=0$ implies an infinite slope of $\partial S / \partial T(T \rightarrow 0)$. This scenario was proposed by theoretical models $[3$ and claimed to correspond to experimental results 2 dismissing some thermodynamic postulates.

We will analyze now the existence of an eventual upper limit for $\partial S / \partial T(T \rightarrow 0)$ and, in the following subsection, the thermodynamic consequences of the proposed divergencies at $T \rightarrow 0$. In Fig. 11 we have collected the low temperature $S_{m}(T)$ dependencies extracted from a number of Ce system showing the highest values of $C_{m} / T \geq 3 \mathrm{~J} / \mathrm{molK}^{2}$ for a doublet GS $(N=2)$ independently that they order or not. This comparison includes the well known $\mathrm{CeCu}_{6-x} \mathrm{Au}_{x}$ 27. and $\mathrm{CeCu}_{6-x} \mathrm{Ag}_{x}$ 65] Ce-lattices. Also the diluted $\left(\mathrm{Ce}_{0.1} \mathrm{La}_{0.9}\right)$ TiGe [66] is included to confirm that this limit is related to thermodynamic properties independently of a lattice configuration. Other Ce diluted systems, like $\left(\mathrm{Ce}_{1-x} \mathrm{La}_{x}\right) \mathrm{Pt}_{3} \mathrm{Si}$ 67, also exhibits similar high values for $0.8 \leq x \leq 0.95$.

As it can be seen, all experimental results show a sort of envelope curve described by the $S_{m}(T)$ dependence of $\mathrm{CePd}_{3} \mathrm{~B}$ 68. To our knowledge, the record of low temperature entropy was extracted from the Cediluted $\left(\mathrm{Ce}_{0.03} \mathrm{La}_{0.97}\right) \mathrm{B}_{6}$ 69 which exceeds the low temperature $S_{m}(T)$ values of $\mathrm{CePd}_{3} \mathrm{~B}$ even after normalized by $R \operatorname{Ln} 4$ (remind that $\mathrm{CeB}_{6}$ has a $\mathrm{N}=4$ GS). Among the Ce-lattice systems, $\mathrm{CeNi}_{9} \mathrm{Ge}_{4}$ shows a very high value: $C_{m} / T(T \rightarrow 0)=5.2 \mathrm{~J} / \mathrm{molK}^{2}[20$, because its GS is composed by the contribution of two hybridized doublets with a Kondo temperature equivalent to the extremely small $\mathrm{CF}$ splitting $(\approx 10 \mathrm{~K})$. Also in this case the corresponding normalization entropy is $R \operatorname{Ln} 4$.

From these experimental evidences we conclude that there is an upper limit for $\partial S / \partial T(T \rightarrow 0) \approx 4 \mathrm{~J} / \mathrm{molK}^{2}$ for $N=2$ GS in Ce systems and consequently a lower limit for the Kondo temperature evaluation based on the thermal dependence of the entropy. The question arises whether this is a physical limit for $T_{K}$ or simply a limit for the application of theoretical models.

\section{B. Divergencies at $T \rightarrow 0$}

Two types of divergencies for thermodynamic parameters were proposed to account for the low energy excitations in NFL systems [2, one described by a power law like $C_{m} / T \propto T^{q}$ and the other by a logarithmic dependence like $C_{m} / T \propto-\ln \left(T / T_{0}\right)$. Details concerning the range of applicability of different models exceed the scope of this phenomenological work and can be found in many interesting review articles [3, 21, 23, 60, 70].

Most of Ce systems obeying a $C_{m}(T) / T$ power law dependence are described by non fractional values of the exponent $q$, with scarce coincidence with theoretical predictions devoted to systems with short range order parameter fluctuations [23, 70]. Divergent power laws imply a non analytical singularity at $T=0$ which is in conflict with 
the third law of thermodynamics. In agreement with thermodynamics, actual specific heat results show a systematic tendency to saturation of $C_{m}(T) / T=\gamma_{T}$ at the low temperature limit, which is well described by an heuristic modified power law $\gamma_{T}=G /\left(T^{q}+A\right)$ [25. In that formula $A$ represents an energy scale below which the third law constraint of $C_{m} \rightarrow 0$ with $T \rightarrow 0$ [26] becomes dominant. That parameter also allows to evaluate the temperature of the crossover between NFL and FL regimes since the later does not diverge at $T \rightarrow 0$. The $T=0$ limit of that formula is obtained computing the limit of $\gamma_{T \rightarrow 0}=G / A$. In the case of the $\mathrm{CePd}_{3} \mathrm{~B}$ compound, proposed in Fig. 11 as the phenomenological envelope curve, the fitting function obtained between 0.5 and $4 \mathrm{~K}$, is $C_{m} / T=4.3 /\left(T^{1.8}+1.1\right)$ which results in a $\gamma_{T \rightarrow 0} \approx 4 \mathrm{~J} / \mathrm{molK}^{2}$ value.

The other usual divergence observed in NFL systems has a logarithmic character. Besides the fact that this type of algebraic divergence does not imply a singularity at $T=0$, even the system showing the highest $C_{m} / T$ values observed at low temperature in $\mathrm{CeCu}_{5.9} \mathrm{Au}_{0.1}$ [60] (included in Fig. 11) does not exceed the $S_{m}(T)$ curve represented by $\mathrm{CePd}_{3} \mathrm{~B}$ significantly. The comparative study performed normalizing the temperature with the respective energy scale $T_{0}$ as $t=T / T_{0}\left[59\right.$ shows that a universal function $C_{m} / t=-D \log (t)+E_{0} \times T_{0}$ describes all analyzed compounds, with $D=7.2 \mathrm{~J} / \mathrm{molK}$ and $E_{0}$ accounting for any eventual high temperature contribution (which is zero for $\mathrm{CeCu}_{5.9} \mathrm{Au}_{0.1}$ ). Once subtracted the non logarithmic contribution $E_{0} \times T_{0}$ the computed entropy does not exceed $60 \%$ of the $R L n 2$ value.

Interestingly, there is a common feature in systems showing a $C_{m} / T \propto-\operatorname{Ln}\left(T / T_{0}\right)$ divergency before to reach the critical point, that is the specific heat jump transformed into a kink. Such an anomaly is well illustrated by $\mathrm{CeCu}_{5.8} \mathrm{Au}_{0.2}$ and $\mathrm{CeIn}_{2.55} \mathrm{Sn}_{0.45}$ samples [25], which requires of the temperature derivative of $C_{m}$ to observe a a discontinuity at $T_{m}$ as shown in the inset of Fig. 8. That feature is also observed in magnetic field driven phase boundary of $\mathrm{Sr}_{3} \mathrm{Ru}_{2} \mathrm{O}_{7}$ [1]. Based on the fact that in $\mathrm{CeCu}_{6-x} \mathrm{Au}_{x}$ the low energy excitations were recognized from neutron scattering measurement to arise from magnetic fluctuations with an effective dimensionality smaller than three [72, one may infer that the mentioned cusp reflects the low dimensionality of the order parameter in this quantum critical region. Analyzing the $C_{m}(T)$ dependence around that transition, its symmetry respect to $T_{M}$ reminds those observed in other Ce compounds in similar conditions, i.e. with $T_{M} \leq 2.5 \mathrm{~K}$ [47.

Thermal expansion also provides phenomenological information for this scenario from measurements performed on $\mathrm{Ce}\left(\mathrm{Pd}_{1-x} \mathrm{Cu}_{x}\right)_{2} \mathrm{Si}_{2}$ in two perpendicular crystalline directions: $\alpha_{c}$ and $\alpha_{a b}$ [73. In this case one observes that, once the $\Delta C_{m}\left(T_{N}\right)$ jump (characteristic for a 3-D mean field like transition) is smeared by the magnetic decoupling between Ce planes, the corresponding $\alpha(T)$ discontinuity transforms into a cusp before to vanish as a function of $x$. The outstanding aspect of this change is that it occurs at different concentrations depending on the measured direction. While in 'c' direction the evolution goes hand by hand with the specific heat jump vanishing at $x=0.2$, along the 'ab' plane it holds up to $x=0.3$ but transforming into a cusp at $T \approx 1 \mathrm{~K}$ as expected for a lower dimensionality order parameter, see Fig.10 in Ref.[12].

\section{Consequences on the lower $T_{K}$ limit determination}

Further consequences arise from thermodynamical constraints imposed by the third law concerning the evaluation of the Kondo temperature from the entropy, e.g. $S_{m}\left(T_{K}\right)=2 / 3 R L n 2$ [74]. If there is an upper limit for the $\partial S_{m} / \partial T$ derivative, a consequent lower limit occurs on the value of $T_{K}$ extracted from $S_{m}(T)$. From the envelope curve proposed in Fig. 11 such a limit would be $\approx 1.3 \mathrm{~K}$. Similar situation occurs with models extracting $T_{K}$ from the specific heat jump at $T_{N}$ as $T_{K} / T_{N} \propto \Delta C_{0} / \Delta C_{m}$ [75], being $\Delta C_{0}=1.5 R$ the reference value from mean field calculation of $\Delta C_{m}$ for a doublet GS. In this procedure the fixed 1.5R value contradicts the constraint imposed by the mentioned law of corresponding states which requires that $\Delta C_{m} \rightarrow 0$ as $T_{N} \rightarrow 0$ affecting the application of the $\Delta C_{0} / \Delta C_{m}$ ratio for a $T_{K}$ evaluation. Another current criterion to evaluate the Kondo temperature is to compute $T_{K} \propto 1 / \gamma_{0}$ [15. Also in this procedure the minimum value of $T_{K}$ is limited by the empirical maximum of $\lim _{T \rightarrow 0} \partial S_{m} / \partial T=\gamma_{0}$ observed in Ce systems. Whether this low $T_{K}$ limit arising from thermodynamic conditions on $\gamma_{0}$ and $\Delta C_{m}$ is intrinsic to the Kondo effect itself is an open question. Theoretical models remark the possibility of a quenching of Kondo effect approaching a QCP [21, 22, but not based on thermodynamic constraints. In any case, these considerations warn on the accurate application of theoretical models not accounting for thermodynamical constraints on real systems.

\section{CONCLUSIONS}

This comparative analysis of the low temperature properties of Ce-magnetic systems show the power of thermodynamic parameters in recognizing different types behaviors, in particular the not fully profited information extracted from the entropy at $T \rightarrow 0$. Moreover, the third law of thermodynamic provides universal sine quibus non conditions for real systems to approach a zero temperature QCP, independently of a priori model hypothesis. It is important to 
distinguish between candidates to present QCP and other with alternative behaviors because new relevant physical phenomena might be missed due to a misleading low temperature extrapolation.

A significant amount of experimental evidences were analyzed at the light of these criteria, which allow to conclude that at least three types of phase diagrams can be clearly distinguished. Depending on the behavior of the $T_{M O}$ phase boundaries, those phase diagrams can be sorted as follows: i) those where the phase transition fulfills the conditions to be driven to $T=0$, ii) those whose phase boundaries vanish at finite temperature because their MO degrees of freedom are progressively transferred to a non magnetic component, and iii) those ending in a critical point at finite temperature.

In the first case the possibility to reach a QCP is supported by the continuous decrease of the $S_{M O}$ entropy, which extrapolates to zero as $T_{M O} \rightarrow 0$. Despite of its monotonous decrease, the phase boundary driven by alloying Celigands shows a change of curvature at $x=x_{0}$. This behavior is attributed to a change from classical to quantum critical of regime since beyond that concentration quantum fluctuations seem to dominate the scenario. Strikingly, such a change occurs at similar temperature energy $T^{C R} \approx 2.2 \pm 0.3 \mathrm{~K}$ in all studied systems, and below that temperature a tendency to saturation of the maximum of $C_{m}(T) / T$ arises as a distinctive characteristic according to a law of corresponding states for a $T=0$ critical point.

A number of distinctive properties were highlighted by a detailed analysis of the thermal properties of the exemplary system $\mathrm{CeIn}_{3-x} \mathrm{Sn}_{x}$ : i) there is an anomalous reduction of about $40 \%$ of the entropy respect to reference value $R \ln 2$ expected for a doublet GS. This missed entropy can be regarded as a zero temperature remanent entropy, ii) it can be quantitatively demonstrated that, contrary to current suppositions, the reduction of $S_{M O}$ as $T_{M O} \rightarrow 0$ is not transferred to the paramagnetic phase, and iii) at the critical region defined by $x=x_{0}$ and $T=T^{C R}$ there is a systematic presence of a first order transition and beyond that point the phase boundary $T_{M}(x)$ changes its nature, manifested in a strong dependence on magnetic field and a $C_{m}(T)$ jump only observed in its temperature derivative.

No evidences for $T \rightarrow 0$ divergencies are observed in real systems, instead a progressive saturation of $C_{m} / T$ is observed in those cases described by a power law a finite temperature. Neither those systems with $\propto-\operatorname{Ln}(T / 0)$ dependence exceed the empirical upper limit of $\gamma_{0} \approx 4 \mathrm{~J} / \mathrm{molK}^{2}$ for Ce systems with doublet GS.

The second type of $S_{M O}\left(T_{M O}\right)$ behavior is currently observed in pressure driven phase boundaries. In this case, specific heat results indicate that the phase boundary itself vanishes because of a progressive transference of degrees of freedom to the non-magnetic component occurring at $T \geq 2 \mathrm{~K}$. Despite of the formation of a superconductive phase their magnetic phase boundaries do not reach that transition because it occurs below the $2 \mathrm{~K}$ threshold. This type of behavior is also observed in Ce-ligand alloyed system, but there the occurrence of superconductivity is unlikely because of allying effects.

The distinct characteristic of the third class of phase diagrams is given by the fact that the $C_{m}\left(T_{N}\right)$ maxima values are found to be constant instead of $C_{m} / T$ like in the first group. In this case the entropy accumulation as $T_{N}$ decreases makes the phase boundary to end at a finite temperature critical point. There, a first order transition drops $S_{M O}$ down to 0 . This peculiar scenario was detected in a system driven by Ce-ligand composition and confirmed by a well know U compound driven by magnetic field. Notably both systems coincide in their $S_{M O}$ values.

To our knowledge, many of these experimental observations were not predicted by current models focused into the physics of QCPs. This is probably due to the difficulty of a generic treatment of thermodynamic parameters like entropy or the specific heat jump in a region dominated by a complex spectra of quantum excitations.

\section{ACKNOWLEDGMENTS}

The author acknowledges A. Eichler, M. Jaime, G. Knebel, M. Deppe and R. Kuechler for allowing to access to experimental data, to S. Grigera, U. Karahasanovic and C. Proetto for illustrative discussions. The studies on $\mathrm{CeIn}_{3-x} \mathrm{Sn}_{x}$ and $\mathrm{CePd}_{1-x} \mathrm{Rh}_{x}$ systems were carried in collaboration with C. Geibel supported by DAAD, Alexander von Humboldt Fundation, PICTP-2007-0812 and SeCyT-UNCuyo 06/C326 projects. Experimental contribution of M. Gómez Berisso and P. Pedrazzini is also acknowledged.

[1] T. Vojta; Ann. Phys. (Leipzig) 9 (2000) 403.

[2] G.R. Stewart, Rev. Mod. Phys. 73 (2001) 797.

[3] H.v. Löhneysen, A. Rosch, M. Vojta, P. Wölfle; Rev. Mod. Phys. 79 (2007) 1015.

[4] A. Iandelli and E. Botti; Rend. Accad. Nac. Lincei, 25 (1937) 129.

[5] D.C. Koskenmaki and K.A. Gschneidner Jr; in Handbook for Physics and Chemistry of Rare Earths, edited by K.A. Gschneidner Jr. and L. Eyring (North Holland Pub. Co., Amsterdam, 1978), Vol 1, Chap. 4.

[6] A.R. Mackintosh; Physica 130B (1985) 112. 
[7] C. Probst and J. Wittig, in Handbook for Physics and Chemistry of Rare Earths, edited by K.A. Gschneidner Jr. and L. Eyring (North Holland Pub. Co., Amsterdam, 1978), Vol 1, Chap. 10. See Fig. 10.8.

[8] A.T. Holmes, D. Jaccard, K. Miyake; Rev. B 69 (2004) 024508.

[9] P.G. Pagliuso, R. Movshovich, A.D. Bianchi, M. Nicklas, N.O. Moreno, J.D. Thompson, M.F. Hundley, J.L. Serrao and Z. Fisk; Physica B 312-313 (2002) 129.

[10] J.G. Sereni and O. Trovarelli, Phill. Mag. Lett. 68 (1993) 231.

[11] S. Doniach, Physica B $\underline{91}$ (1977) 231 and M. Lavagna, C. Lacroix and M. Cyrot, Phys. Lett. 90A (1982) 210.

[12] J.G. Sereni, J. Phys. Soc. Japan, 70 (2001) 2139.

[13] J.G. Sereni; J. Alloys and Compounds 207/208 (1994) 229.

[14] A change in the chemical potential $\mu$ implies a significant modification in the Fermi energy of the band because of the variation of the number of electrons $n$ (c.f. Pd has one more electron than Rh, with similar atomic volume). On the other hand, a structural pressure $p_{s t r}$ is originated in the different atomic volume $\Delta V$ (c.f. $\mathrm{Pd}$ and $\mathrm{Ni}$, with similar electronic structure) of the Ce-ligand atoms without. In the former case the thermodynamical relations can be written through the Gibbs energy like: $\mu=\partial G / \partial n$ and $P_{s t r}=\partial G / \partial \Delta V$ for the latter.

[15] See e.g. V.T. Rajan, Phys. Rev. Lett. 51 (1983) 308.

[16] These types of GS are currently identified as related to small (LM) or large (ItM) Fermi-surfaces, see for example 21.

[17] J.G. Sereni; Physica B 215 (1995) 273.

[18] B. Coqbiln and J.R. Schrieffer, Phys. Rev. 185 (1969) 847.

[19] M. Deppe, N.C-Caroca, S. Hartmann, N. Oesler, C. Geibel, J. Phys.: Condens. Matter 21 (2009) 206001.

[20] U. Killer, E-W. Scheidt, W. Scherer, H. Michor, J. Sereni, Th. Pruschke and S. Kehrein, Phys. Rev. Lett. 93 (2004) 216404.

[21] Q. Si and F. Steglich, Science 329 (2010) 1161.

[22] P.Coleman and A.J. Schofield, Nature 433 (2005) 226.

[23] M.A. Continentino, Braz. J. Phys. 41 (2011) 201.

[24] Strictly, long range of short range magnetic order are not different in the following dicussion becauseit is base on the entropy (i.e. degress of freedom) involved into the decreasing magneic interactions.

[25] J.G. Sereni; J. Low Temp. Phys. 147 (2007) 179.

[26] See for example: H.B. Callen, in Thermodynamics and an Introduction to Thermostatistics (2nd ed.), New York: John Wiley \& Sons, 1985.

[27] H.v. Löhenysen, T. Pietrus, G. Portisch, A. Schröder, H.G. Schlager, M. Sieck, T. Trappmann, Phys. Rev. Lett. 72 (1994) 3262.

[28] G. Knebel, M. Brando, J. Hemberger, M. Nicklas, W. Trinkl, A Loidl, Phys. Rev. B 59 (1999) 12390.

[29] E. Brück, H. Nakotte, K. Bakker, F.R. de boer, P.F. de Chatel, J.-Y. Li, J.P.Kuang, F.-M. Yang, J. Alloys and Comps. 200 (1993) 79.

[30] T. Kirsch, A. Eichler, P. Morin, U. Welp; Z. Phys. B - Cond. Matter 86 (1992) 83.

[31] see for example: N.D. Mathur et al., Nature 394 (1998) 39 and G. Knebel et al., Phys. Rev. B 65 (2001) 024425.

[32] J. Peyrard; PhD Thesis, University of Grenoble, 1980.

[33] H. Neemann, A. Eichler, S. Suellow, J.A. Mydosh; Acta Phys. Polon. B 34 (2003) 1085.

[34] G. Knebel, D. Braithwaite, P.C. Canfield, G. Laperot, J. Flouquet; High Pressure Reasearch 22 (2002) 167.

[35] I. Umehara, M. Hedo, F. Tomioka, Y. Uwatoko, J. Phys. Soc. Jpn. 76 (2007) Suppl. A, 206.

[36] F. Bouquet, Y. Wang, H. Wilhelm, D. Jaccard, A. Junod; Sol. State Commun. 113 (2000) 367.

[37] S. Kawasaki, M. Yashima, Y. Kitaoka, K. Takeda, K. Shimizu, Y. Oishi, M. Takata, T. C. Kobayashi, H. Harima, S. Araki, H. Shishido, R. Settai, Y. O-nuki, Phys. Rev. B 77 (2008) 064508.

[38] S. Demuer, D. Jaccard, I. Sheikin, S. Raymond, B. Salce, J. Thomasson, D. Braithwaite, J. Flouquet; J. Phys. Condensed Matter 13 (2001) 9335.

[39] J.G. Sereni, Rev. Esp. de Fsica, 13 (1999) 25.

[40] J. Larrea, M.B. Fontes, A.D. Alvarenga, E.M. Baggio-Saitovitch, T. Burghardt, A. Eichler, M.A. Continentino; Phys. Rev. B 72 (2005) 035129.

[41] See for example J. Flouquet, D. Aoki, F. Bourdarot, F. Hardy, E. Hassinger, G. Knebel, T.D. Matsuda, C. Meingast, C. Paulsen, V. Taurfour, J. Physics: Conference Series 273 (2010) 012001.

[42] P. Haen, F. Lapierre, P. Lejay, T. Jaworska-Golab, C. Sekine, S. de Brion; Acta Phys. Pol. B, 34 (2003) 1047.

[43] M.B. Maple, S.E. Lambert, M.S. Torikachvili, K.N. Yang, J.W. Allen, B.B. Pate, I. Lindau; J. Less Comm. Metals 111 (1985) 239.

[44] J.G. Sereni, G. Schmerber, A. Braghta, B. Chevalier, J.P. Kappler; arXiv 1103.0190 cond-mat, 1 March 2011.

[45] M. Jaime, K.H. Kim, J. Guillermo, S. McCall, J.A. Mydosh; Phys. Rev. Lett., 89 (2002) 287201.

[46] J.G. Sereni, T. Westerkamp, R. Küchler, N. C.-Cananles, P. Gegenwart, C. Geibel; Phys. Rev. B 75 (2007) 024432.

[47] J.G. Sereni; Physica B 398 (2007) 412.

[48] P. Pedrazzini, M.Gómez Berisso, N. Caroca Canales, M. Deppe, C. Geibel ans J.G. Sereni; Eur. Phys. J. B 38 (2004) 445.

[49] O.Trovarelli, M. Weiden, R. M.-Reisener, M. G.-Berisso, P. Gegenward, M. Deppe, C. Geibel, J.G. Sereni and F. Steglich, Phys. Rev. B 56 (1997) 678.

[50] M. Deppe, P. Pedrazzini, N. Caroca-Canales, C. Geibel, J.G. Sereni, Physica B 378 (2006) 96.

[51] A. Loidl, A. Krimmel, K. Knorr, G. Sparn, M. Lang, C. Geibel, S. Horn, A. Grauel, F. Steglich, B. Welslau, N. Grewe, H. Nakotte, F. R. de Boer, A. P. Murani; Annalen der Physik 504 (1992) 78.

[52] Remind that Nordheim criterion [53] for disordered alloys propose a $\rho_{0} \propto x(1-x)$ distribution, which is centered at $x=0.5$. An asymmetrical $x$ dependence is expected for alloyed elements with different electronic orbitals, including $s-d$ 
electronic transition.

[53] See for example N.F. Mott and H. Jones, in The theory of metals and alloys, Dover Pub. Inc. N.Y. 1958.

[54] T. Rus, H. Wilhelm, O. Stockert, T. Lühmann, N. C- Canales, J.G. Sereni, C. Geibel and F. Stelich, Physica B 359-361 (2005) 62.

[55] A.V. Silhanek, T. Ebihara, N. Harrison, M. Jaime, K. Tezuka, V. Fanelli, C.D. Batista; Phys. Rev. Lett. 96 (2006) 206401.

[56] U. Kasahasanovic, F. Krüger, A.G. Green; arXiv: 1201.3616 [cond-mat] 24 Jan 2012.

[57] See for example: A. B. Pippard, in Elements of Classical Thermodynamics for Advanced Students of Physics, Cambridge University Press, 1957.

[58] see for example: J.M. Lawrance and S.M. Shapiro; Phys. Rev. B 22 (1980) 4379 and W. Gross, K. Korm, A.P. Murani, K.H.J. Buschow, Z. Phys. B 37 (1980) 123.

[59] J.G. Sereni, C. Geibel, M. G.-Berisso, P. Hellmann, O. Trovarelli and F. Steglich, Physica B 230 (1997) 580.

[60] H.v. Löhneysen; J. Magn. Magn. Mater. 200 (1999) 532.

[61] see for example: C. Domb and A.R. Mienema, "Magnetic Transitions" in Progress in Low Temperature Physics, Ed. by C.J. Gorter, North- Holland Pub.Co., (1964) Vol IV, Ch.6, p.296.

[62] J.G. Sereni; Physica B 354 (2004) 331.

[63] R. Kuechler: PhD Thesis, University of Darmstadt, 2003.

[64] J.P. Abriata and D.E. Laughlin, Prog. Matterial Science 49 (2004) 367.

[65] K. Heuser, E.-W. Scheidt, T. Schreiner, G.R. Stewart Phys. Rev. B Rapid Comm. 58, R15959, (1998)

[66] J. G Sereni, M. G.-Berisso, M. Deppe, N.C.Canales, C. Geibel; Phys. Stat. Solidi (b), 247 (2010) 707.

[67] J.S. Kim, D.J. Nixson, D.J. Burnette, G.R. Stewart; J. Low Temp. Phys. 147 (2007) 135.

[68] J.G. Sereni, G. Nieva, J. Kappler, M. Besnus, A. Meyer, J. Physics F (Metal Phys.) 16 (1986) 435.

[69] N. Sato, M. Takahashi, T. Kashima, K. Sugiyama, M. Date, T. Satoh, T. Kasuya; J. Magn. Magn. Mat. 52 (1985) 250.

[70] E. Miranda and V. Dobrosavljević; Rep. Prog. Phys. 68 (2005) 2337.

[71] A.W. Rost, S. A. Grigera, J.A.N. Bruin, R.S. Perry, D. Tian, S. Raghu, S.A. Kivelson, A.P. Mackenzie, PNAS 108 (2011) 16549.

[72] H.v. Löhneysen, A. Schroeder, O. Stockert, J. Alloys and Comps. 303-304

[73] F.Kromer; PhD Thesis, Technical University Dresen, 2000.

[74] See for example H.-U. Desgranges and K.D. Schotte, Physics Letters 91A (1982) 240.

[75] see for example A. Braghta, PhD Thesis, Univ. L. Pasteur, Strasbourg, 1989. 